\title{
Further Investigation of Stress Intensity Factor Solutions for Similar and Dissimilar Welds in Lap-Shear Specimens under Clamped Loading Conditions
}

\author{
Shin-Jang Sung and Jwo Pan ${ }^{1}$ \\ Department of Mechanical Engineering \\ The University of Michigan \\ Ann Arbor, MI, 48109, USA
}

August 3, 2016

\begin{abstract}
In this paper, finite element analyses are employed to obtain the stress intensity factor solutions for similar and dissimilar welds in lap-shear specimens under pinned and clamped loading conditions. Analytical solutions for welds in lap-shear specimens under clamped loading conditions are then derived based on the energy release rate and the structural stress approaches. Both computational and analytical solutions indicate that the clamped loading reduces the stress intensity factor solutions for a given specimen geometry by about $7 \%$ for similar welds and by about $20 \%$ for dissimilar aluminum/steel and magnesium/steel welds, and results in higher fatigue lives of the welds.
\end{abstract}

Keywords: similar weld; dissimilar weld; lap-shear specimen; stress intensity factor; clamped loading

${ }^{1}$ Corresponding author. Tel.:+1-734-764-9404; fax:+1-734-647-3170

Email address: jwo@umich.edu (Jwo Pan) 


\section{Nomenclature}

A, B, C, D, E locations of a beam

$b \quad$ specimen width

$C_{u} \quad$ corrective term for the upper sheet due to clamped loading (function of $\delta$, $\eta, r / L)$

$C_{l} \quad$ corrective term for the lower sheet due to clamped loading (function of $\delta$, $\eta, r / L)$

$d x \quad$ increase of the crack length

$d U \quad$ increase of the elastic strain energy

$E_{u} \quad$ Young's modulus of the upper sheet

$E_{l} \quad$ Young's modulus of the lower sheet

$E_{u}^{\prime} \quad$ effective Young's modulus of the upper sheet under plane strain conditions

$E_{l}^{\prime} \quad$ effective Young's modulus of the lower sheet under plane strain conditions

$f_{1}, f_{2} \quad$ normalized stress intensity factor solutions for an interface crack

$f_{I}, f_{I I} \quad$ normalized stress intensity factor solutions for similar sheet materials

$f_{e} \quad$ normalized effective stress intensity factor

F lap-shear load

$G_{u} \quad$ shear modulus of the upper sheet

$G_{l} \quad$ shear modulus of the lower sheet

$G \quad$ energy release rate

I moment of inertia 
$\mathbf{K}\left(=K_{1}+i K_{2}\right) \quad$ complex stress intensity factor for an interface crack

$\mathbf{K}^{\mathrm{A}}\left(=K_{1}^{A}+i K_{2}^{A}\right) \quad$ complex stress intensity factor for an interface crack obtained from

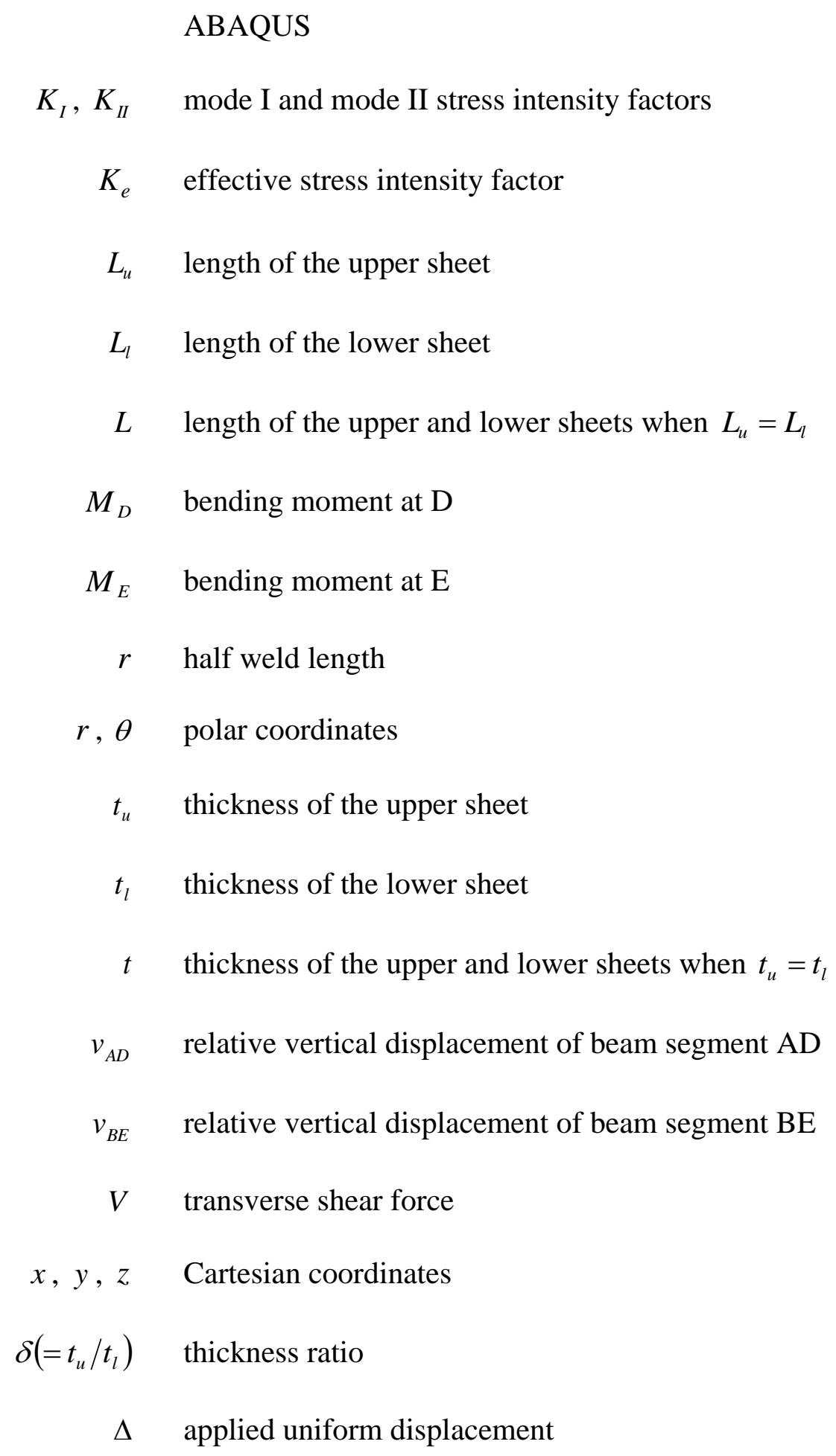

$K_{I}, K_{I I} \quad$ mode I and mode II stress intensity factors

$K_{e} \quad$ effective stress intensity factor

$L_{u} \quad$ length of the upper sheet

$L_{l} \quad$ length of the lower sheet

$L \quad$ length of the upper and lower sheets when $L_{u}=L_{l}$

$M_{D} \quad$ bending moment at $\mathrm{D}$

$M_{E} \quad$ bending moment at $\mathrm{E}$

$r \quad$ half weld length

$r, \theta \quad$ polar coordinates

$t_{u} \quad$ thickness of the upper sheet

$t_{l} \quad$ thickness of the lower sheet

$t \quad$ thickness of the upper and lower sheets when $t_{u}=t_{l}$

$v_{A D} \quad$ relative vertical displacement of beam segment $\mathrm{AD}$

$v_{B E} \quad$ relative vertical displacement of beam segment $\mathrm{BE}$

$V \quad$ transverse shear force

$x, y, z \quad$ Cartesian coordinates

$\delta\left(=t_{u} / t_{l}\right) \quad$ thickness ratio

$\Delta \quad$ applied uniform displacement 


$$
\begin{array}{cl}
\eta_{u}\left(=E_{u}^{\prime} / E_{l}^{\prime}\right) & \text { bimaterial constant } \\
\theta_{A D} & \text { relative rotation of beam segment AD } \\
\theta_{B E} & \text { relative rotation of beam segment BE } \\
\kappa(=3-4 v) & \text { coefficient } \\
\lambda & \text { function of } r / L \text { as in Equation (42) } \\
v_{u} & \text { Poisson's ratio of the upper sheet } \\
v_{l} & \text { Poisson's ratio of the lower sheet } \\
\sigma_{y}, \tau_{x y} & \text { opening and shear stresses in the Cartesian coordinate system } \\
\omega & \text { angular quantity that can be found in [4] and [5] }
\end{array}
$$

Superscripts and Superscripts

A, B, C, D, E locations of a beam

A solutions obtained from ABAQUS

$i, o \quad$ inner and outer surfaces of the sheets

$p, c \quad$ pinned and clamped loading conditions

$u, l \quad$ upper and lower sheets 


\section{Introduction}

Automotive sheet components are commonly joined by spot welds such as resistance spot welds and continuous welds such as laser welds. The fatigue strengths of these welds are important to the durability of vehicles. For improving fuel economy as well as addressing environmental issues, the automotive industry is investigating ways to increase the use of lightweight materials such as magnesium and aluminum alloys. When aluminum and magnesium alloys are considered for various automotive sheet components that require attaching to steel sheet components, research on the fatigue strengths of dissimilar welds becomes important and necessary. Lap-shear or tensile-shear specimens are most commonly used to evaluate the fracture and fatigue strengths of welds. When the plastic zones near the fronts of the pre-existing cracks of the welds are small in the specimens under cyclic loading conditions due to the small applied cyclic loads, the stress intensity factor solutions of the linear elastic fracture mechanics are useful to correlate with the fatigue lives of the welds in the specimens. Therefore, accurate stress intensity factor solutions are important and necessary to correlate well with or predict the fatigue lives of the welds in the specimens.

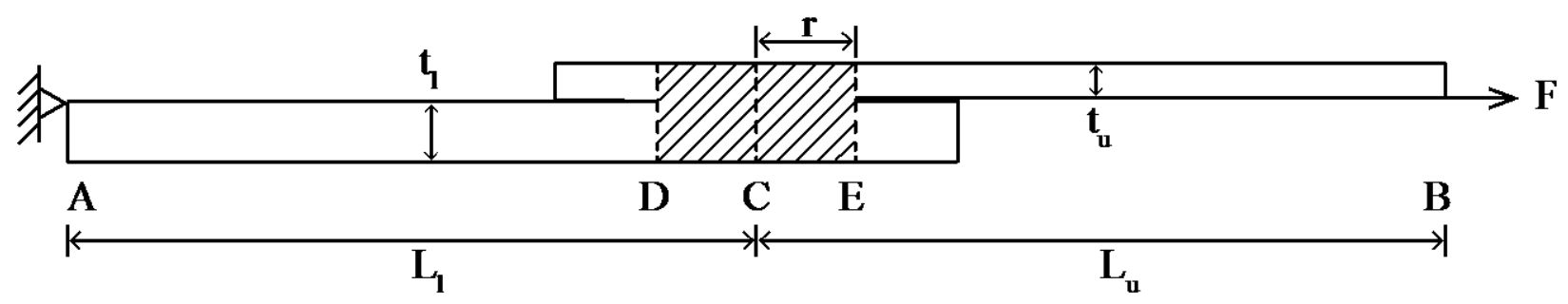

Figure 1. A lap-shear specimen under pinned loading conditions. 
For testing the welds in lap-shear specimens, the fixture can be made such that the specimens are subjected to pinned loading conditions as schematically shown in Figure 1. In this paper, we investigate the type of the lap-shear specimen that is cut from two sheets welded with a continuous weld as that in Sripichai et al. [1] such that the specimen can be treated as a twodimensional plane strain problem as schematically shown Figure 1. In the figure, the weld between the upper and lower sheets are shown as the shaded area between D and E. A set of doublers or spacers is usually needed to be used to align the specimen during testing. Here, the lap-shear specimen is investigated under the idealized pinned loading conditions. As shown in Figure 1, the displacements of the upper surface of the left edge of the lower sheet are constrained. A lap-shear load $F$ is applied at the lower surface of the right edge of the upper sheet. In the figure, $t_{u}$ and $t_{l}$ represent the upper and lower sheet thicknesses, respectively, $r$ represents the half weld length, and $L_{u}$ and $L_{l}$ represent the lengths $\mathrm{BC}$ and $\mathrm{AC}$ of the upper and lower sheets, respectively. The stress intensity factor solutions were obtained for the welds in lap-shear specimens of equal sheet thickness under pinned loading conditions by the finite element analyses for different ratios of the weld length to sheet thickness in Sripichai et al. [1]. The results of the finite element analyses in Sripichai et al. [1] showed that the analytical stress intensity factor solutions derived from the energy release rate approach and the beam theory can be used for the welds in lap-shear specimens with the ratio of the weld length to sheet thickness larger than 4 .

Many welds in lap-shear specimens failed in the kinked fatigue crack growth mode from the main cracks or the pre-existing cracks under cyclic loading conditions. For example, the laser welds in lap-shear specimens of high strength low alloy steel failed in the kinked crack growth failure mode as presented in Asim et al. [2]. The global stress intensity factor solutions for the 
main or the pre-existing crack were used as the reference values for the local stress intensity factor solutions for small kinked cracks as presented in Cotterell and Rice [3]. The global and local stress intensity factor solutions for the pre-existing and kinked cracks based on the finite element analyses were used to estimate the fatigue lives of laser welds in the lap-shear specimens of high strength low alloy steel under pinned loading conditions by Sripichai et al. [1] and Asim et al. [2]. For the welds in lap-shear specimens of dissimilar material and unequal thickness, the analytical solutions can be obtained from the solutions of Zhang [4] and Suo and Hutchinson [5] using the structural stress approach. The stress intensity factor solutions for similar and dissimilar welds in lap-shear specimens of steel, aluminum, magnesium, and copper sheets with equal and unequal thickness under pinned loading conditions were presented and validated selectively by the finite element analyses in Lai and Pan [6].

When the commercial clamped specimen grips are used for testing, the lap-shear specimens with welds are tested under clamped loading conditions, as schematically shown in Figure 2 . It should be mentioned again that a set of doublers or spacers also has to be used to align the specimens for testing under clamped loading conditions. Under clamped loading conditions, the displacements of the left edge are constrained as schematically shown in Figure 2. A uniform displacement $\Delta$ is applied at the right edge. When a lap-shear specimen is subjected to the clamped loading conditions, the bending moments and transverse shear forces due to the clamped loading grips can have effects on the stress states near the weld and, consequently, on the stress intensity factors for the weld. The effects of the bending moments and the transverse shear forces at the clamped edges on the stress intensity factor solutions and then the fatigue behavior of the welds have not been investigated systematically in the past. 


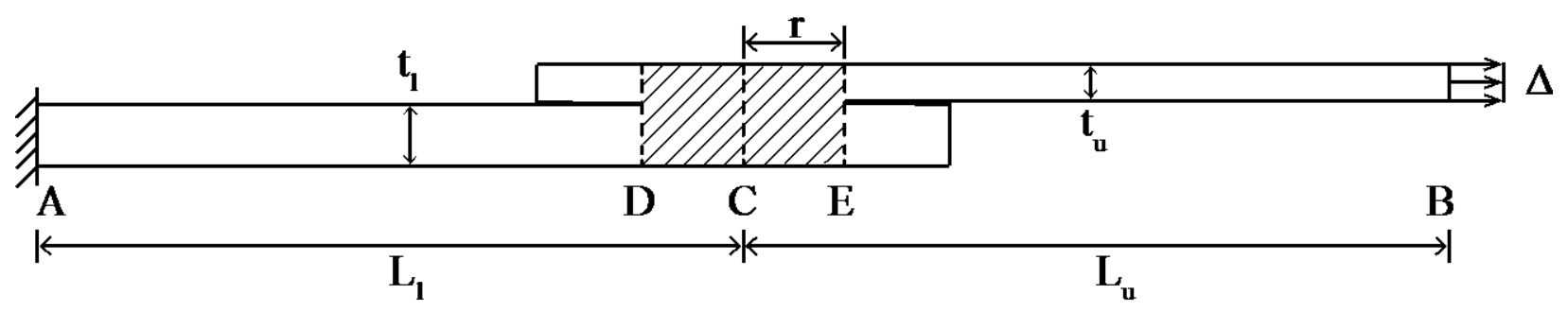

Figure 2. A lap-shear specimen under clamped loading conditions.

Hong et al. [7] examined the failure mode and fatigue behavior of the friction stir spot welds in lap-shear specimens of transformation induced plasticity steel and hot stamped boron steel sheets under clamped loading conditions. Hong et al. [7] derived the analytical solutions for the bending moments and the transverse shear forces at the clamped edges for the lap-shear specimens based on the beam theory. They then used the analytical solutions to obtain the stress intensity factor solutions at the critical locations of the spot welds and estimated the fatigue lives of the spot welds based on the corresponding local stress intensity factor solutions for the kinked cracks emanating from the pre-existing cracks around the welds. However, the effects of the bending moments and transverse shear forces at the loading edges on the stress intensity factor solutions for the spot welds were not explored in Hong et al. [7] systematically due to the complex geometry of the spot welds.

Sung and Pan [8] used the finite element analyses to investigate the stress intensity factor solutions for welds in lap-shear specimens of similar material and equal thickness under pinned and clamped loading conditions. They also derived the analytical stress intensity factor solutions based on the beam theory. The computational and analytical solutions indicate that the bending moments and transverse shear forces at the clamped edges can reduce the mode I and II stress intensity factor solutions by about $7 \%$ to $10 \%$. The closed-form analytical stress intensity factor 
solutions were derived based on the continuity of the displacement and slope at the center of the weld as in Hong et al. [7], where the weld is assumed to be small to simplify the closed-form solutions. It should be mentioned that the analytical stress intensity factor solutions for spot welds in lap-shear specimens were obtained from the assumption that the spot welds were idealized as rigid inclusions $[9,10]$. These solutions appear to be quite accurate. Therefore, the welds in lap-shear specimens are assumed as rigid with a finite length in this investigation to explore the effect of this assumption for the welds in lap-shear specimens of similar and dissimilar material.

In this paper, finite element analyses are first employed to obtain the stress intensity factor solutions for similar welds in lap-shear specimens with two thickness ratios and dissimilar welds in lap-shear specimens with one thickness ratio under pinned and clamped loading conditions. The computational stress intensity factor solutions under pinned and clamped loading conditions are then compared. For validation of the computational solutions from the finite element analyses, analytical stress intensity factor solutions are derived based on the beam theory. In order to obtain the closed-form solutions to explore explicitly the effects of the clamped edges on the stress intensity factor solutions, the analytical solutions for the similar welds in the specimens of equal thickness based on the energy release rate approach are examined. For the specimens of dissimilar material and unequal thickness under clamped loading conditions, the analytical stress intensity factor solutions are obtained from the solutions of Zhang [4] and Suo and Hutchinson [5] using the structural stress approach. The analytical stress intensity factor solutions are then compared with the results of the finite element analyses. Finally, the implications of the results on the fatigue life estimations of welds in lap-shear specimens under pinned and clamped loading conditions are discussed. 


\section{Finite Element Analyses of Lap-Shear Specimens}

It is difficult to visualize the effects of the clamped boundary conditions on the stress intensity factor solutions for the similar and dissimilar welds in lap-shear specimens under clamped loading conditions. Therefore, finite element analyses are first used to examine the effects of the boundary conditions on the stress intensity factor solutions for the welds. Twodimensional finite element models for the specimen of equal thickness under pinned and clamped loading conditions are shown in Figures 3 and 4, respectively. The two-dimensional finite element models are based on the lap-shear specimens with friction stir spot welds used in Hong et al. [7] with $t_{u}=t_{l}=t=1.5 \mathrm{~mm}, r=3.8 \mathrm{~mm}$, and $L_{u}=L_{l}=L=55 \mathrm{~mm}$. Note that the upper and lower sheets of the lap-shear specimens used in Hong et al. [7] have different thicknesses. Therefore, the stress intensity factor solutions for the weld in the specimen of unequal thickness will also be investigated. The overlap of the upper and lower sheets is set to $30 \mathrm{~mm}$. The Cartesian $x-y$ coordinate system is also shown in both figures. Due to the small element sizes of the finite element models, the details of the finite elements could not be shown in Figures 3 and 4.

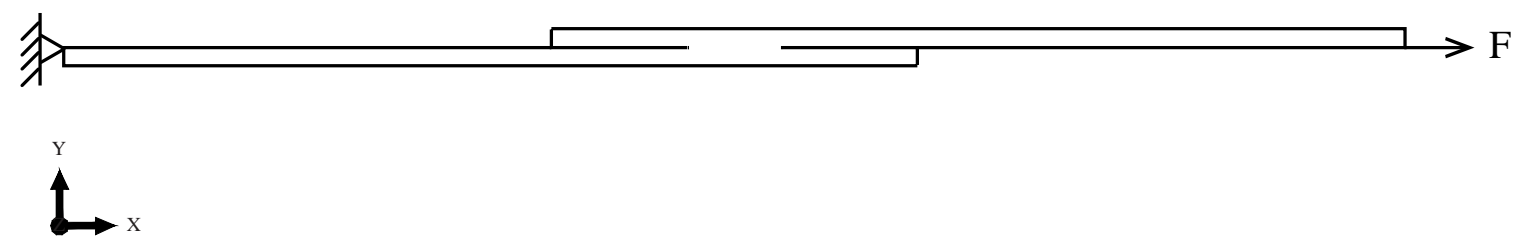

Figure 3. A finite element model for a lap-shear specimen with $\delta=1$ under pinned loading conditions. 


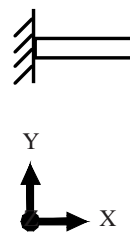

Figure 4. A finite element model for a lap-shear specimen with $\delta=1$ under clamped loading conditions.

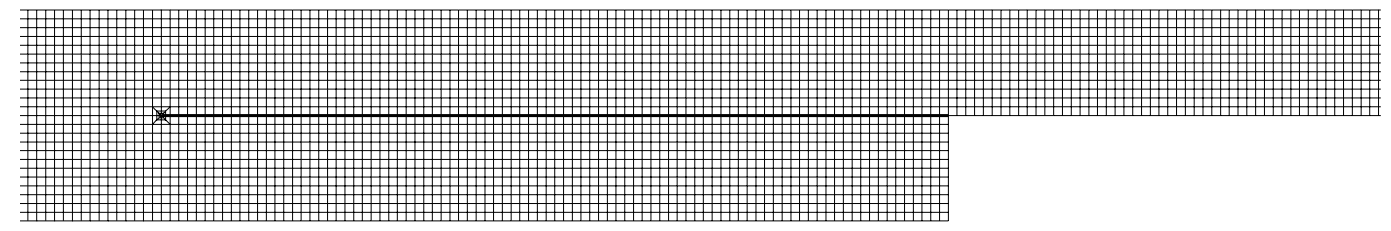

$\stackrel{\mathrm{r}}{\mathrm{A}}$

Figure 5. The mesh near the right crack of the weld in the finite element models.

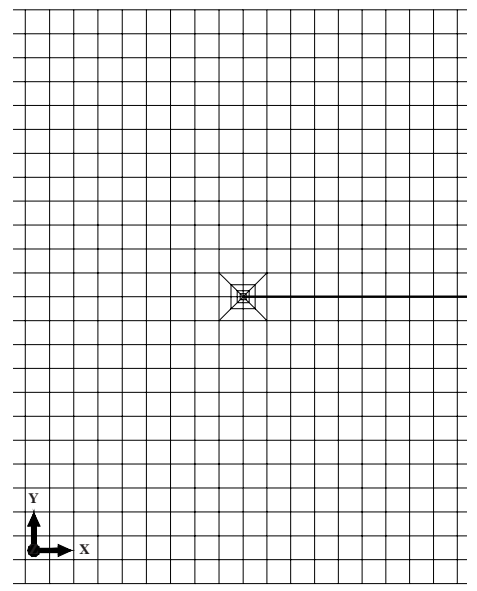

Figure 6. A close-up view of the mesh design near the right crack tip of the weld in the finite element models.

Figure 3 shows the boundary conditions for the pinned loading conditions where the displacements of the upper surface of the left edge of the lower sheet in the $x$ and $y$ directions are constrained. A lap-shear load $F$ is applied at the lower surface of the right edge of the upper sheet in the $x$ direction. Figure 4 shows the boundary conditions for the clamped loading 
conditions where the displacements of the left edge in the $x$ and $y$ directions are constrained. A uniform displacement $\Delta$ is applied to the right edge in the $x$ direction. Figure 5 shows the mesh near the right crack of the weld for both finite element models.

Commercial software ABAQUS was used for the finite element analyses. The plane strain quadratic quadrilateral (CPE8) elements were used. The element size of the nearly uniform meshes of both finite element models is about $0.125 \mathrm{~mm}$. The element size near the crack tip is reduced to $0.015 \mathrm{~mm}$. The number of finite elements for the models under both pinned and clamped loading conditions is 13,496 . The Young's modulus is set to $207 \mathrm{GPa}$ and the Poisson's ratio is set to 0.3 . Quarter point crack-tip elements with collapsed nodes were used to model the $1 / \sqrt{r}$ singularity near the crack tips. Figure 6 shows a close-up view of the mesh near the right crack tip of the weld for both finite element models.

We also conducted a finite element analysis for the weld in a lap-shear specimen of similar material and unequal thickness. The thickness ratio $\delta$ is defined as

$$
\delta=\frac{t_{u}}{t_{l}}
$$

We selected the unequal thickness case of $\delta=0.5$ with $t_{u}=0.75 \mathrm{~mm}$ and $t_{l}=1.5 \mathrm{~mm}$. The same half weld length of $r=3.8 \mathrm{~mm}$ and the specimen length of $L_{u}=L_{l}=L=55 \mathrm{~mm}$ were also used for the unequal thickness case. The finite element models for the case of $\delta=0.5$ are similar to those shown in Figures 3 to 6 and will not be shown here. Finally, finite element analyses of a lap-shear specimen of dissimilar material and equal thickness are also performed. For the first dissimilar weld case, the upper sheet is assumed to be aluminum with the Young's modulus $E_{u}=69 \mathrm{GPa}$ and the Poisson's ratio $v_{u}=0.33$. The lower sheet is assumed to be steel 
with the Young's modulus $E_{l}=207 \mathrm{GPa}$ and the Poisson's ratio $v_{l}=0.3$. For the second dissimilar weld case, the upper sheet is assumed to be magnesium with the Young's modulus $E_{u}=45 \mathrm{GPa}$ and the Poisson's ratio $v_{u}=0.35$. The lower sheet is assumed to be steel with the Young's modulus $E_{l}=207 \mathrm{GPa}$ and the Poisson's ratio $v_{l}=0.3$.

\section{Computational Results}

For convenient presentation of the computational results, the stress intensity factors for the interfacial cracks in this investigation are first defined here. The upper and lower sheets are assumed to be isotropic linear elastic materials. The upper sheet has the Young's modulus $E_{u}$, shear modulus $G_{u}$, and Poisson's ratio $v_{u}$. The lower sheet has the Young's modulus $E_{l}$, shear modulus $G_{l}$, and Poisson's ratio $v_{l}$. The modulus ratio $\eta$ is defined as

$$
\eta=E_{u}^{\prime} / E_{l}^{\prime}
$$

where

$$
E_{u}^{\prime}=E_{u} /\left(1-v_{u}^{2}\right)
$$

and

$$
E_{l}^{\prime}=E_{l} /\left(1-v_{l}^{2}\right)
$$

under plane strain conditions. The asymptotic stress field near the crack tip can be characterized by a complex stress intensity factor $\mathbf{K}\left(=K_{1}+i K_{2}, i=\sqrt{-1}\right)$ (Rice and Sih [11] and Rice [12]). The opening stress $\sigma_{y}$ and the shear stresses $\tau_{x y}$ at a small distance $r$ ahead of the interface crack tip, as referring to the left crack in Figures 3 to 4 with the given Cartesian $x-y$ coordinate system, are characterized by $\mathbf{K}$ as 


$$
\sigma_{y}+i \tau_{x y}=\frac{K_{1}+i K_{2}}{\sqrt{2 \pi r}}\left(\frac{r}{t_{u}}\right)^{i \varepsilon}
$$

where the bimaterial constant $\varepsilon$ is defined as

$$
\varepsilon=\frac{1}{2 \pi} \ln \frac{\kappa_{u} / G_{u}+1 / G_{l}}{\kappa_{l} / G_{l}+1 / G_{u}}
$$

Here, $\kappa_{u}$ and $\kappa_{l}$ are defined as

$$
\kappa_{u}=3-4 v_{u}
$$

and

$$
\kappa_{l}=3-4 v_{l}
$$

In this investigation, $t_{u}$ in Equation (5) is selected as the characteristic length as in Zhang [4].

When the two materials are identical ( $\varepsilon=0$ ), $K_{1}$ and $K_{2}$ in Equation (5) for the interface crack become the conventional stress intensity factors $K_{I}$ and $K_{I I}$, respectively.

In the commercial software ABAQUS, the complex stress intensity factors are defined without the use of the characteristic length $t_{u}$. As indicated in Lai and Pan [6], the complex stress intensity factor $K_{1}^{A}$ and $K_{2}^{A}$ solutions obtained from ABAQUS need to be converted to the complex stress intensity factor $K_{1}$ and $K_{2}$ solutions defined in Equation (5). For dissimilar welds, the stress intensity factor solutions under pinned loading conditions can be expressed in the normalized forms as

$$
\begin{aligned}
& K_{1}=f_{1}^{p} \frac{F}{b \sqrt{t_{u}}} \\
& K_{2}=f_{2}^{p} \frac{F}{b \sqrt{t_{u}}}
\end{aligned}
$$


For convenience, the effective stress intensity factor $K_{e}$ is defined as

$$
K_{e}=\sqrt{K_{1}^{2}+K_{2}^{2}}
$$

Therefore, $K_{e}$ can be written as

$$
K_{e}=f_{e}^{p} \frac{F}{b \sqrt{t_{u}}}
$$

where the superscript "p" represents the pinned loading conditions. Here, $f_{1}^{p}, f_{2}^{p}$, and $f_{e}^{p}$ are the normalized stress intensity factor solutions for the pinned loading conditions. The stress intensity factor $K_{1}, K_{2}$, and $K_{e}$ solutions for a weld in a lap-shear specimen under clamped loading conditions can also be expressed in the normalized forms as

$$
\begin{aligned}
& K_{1}=f_{1}^{c} \frac{F}{b \sqrt{t_{u}}} \\
& K_{2}=f_{2}^{c} \frac{F}{b \sqrt{t_{u}}} \\
& K_{e}=f_{e}^{c} \frac{F}{b \sqrt{t_{u}}}
\end{aligned}
$$

where the superscript "c" represents the clamped loading conditions. Here, $f_{1}^{c}, f_{2}^{c}$, and $f_{e}^{c}$ are the normalized stress intensity factor solutions for the clamped loading conditions.

For similar welds, the stress intensity factor $K_{I}$ and $K_{I I}$ solutions can be expressed in the normalized forms as

$$
\begin{aligned}
& K_{I}=f_{I}^{p} \frac{F}{b \sqrt{t_{u}}} \\
& K_{I I}=f_{I I}^{p} \frac{F}{b \sqrt{t_{u}}}
\end{aligned}
$$


For convenience, the effective stress intensity factor $K_{e}$ can also be defined as

$$
K_{e}=\sqrt{K_{I}^{2}+K_{I I}^{2}}
$$

Therefore, $K_{e}$ can be written as

$$
K_{e}=f_{e}^{p} \frac{F}{b \sqrt{t_{u}}}
$$

where the superscript "p" represents the pinned loading conditions. Here, $f_{I}^{p}, f_{I I}^{p}$, and $f_{e}^{p}$ are the normalized stress intensity factor solutions for the pinned loading conditions. The stress intensity factor $K_{I}, K_{I I}$, and $K_{e}$ solutions for a weld in a lap-shear specimen under clamped loading conditions can also be expressed in the normalized forms as

$$
\begin{aligned}
& K_{I}=f_{I}^{c} \frac{F}{b \sqrt{t_{u}}} \\
& K_{I I}=f_{I I}^{c} \frac{F}{b \sqrt{t_{u}}} \\
& K_{e}=f_{e}^{c} \frac{F}{b \sqrt{t_{u}}}
\end{aligned}
$$

Tables 1 and 2 list the computational normalized stress intensity factor $f_{I}, f_{I I}$, and $f_{e}$ solutions for the similar welds in the specimens with $\delta=1$ and $\delta=0.5$, respectively. The computational results for the similar weld case under pinned loading conditions with $\delta=1$ are in very good agreement with the analytical solution $\left(f_{I}=\sqrt{3 / 2}=0.8660\right.$ and $\left.f_{I I}=1.0000\right)$ given in Sripichai et al. [1] and this agreement indicates the mesh design for the finite element models are reasonably acceptable. Due to the anti-symmetric conditions for $\delta=1$, both the left and right cracks have the same values of the stress intensity factor solutions. For the similar weld 
case with $\delta=0.5$, the stress intensity factor solutions for the right cracks are larger than those for the left crack under both pinned and clamped loading conditions. As listed in the tables, the bending moments and the transverse shear forces at the clamped edges reduce the stress intensity factor solutions by about 5 to $8 \%$ for $\delta=1$ and $\delta=0.5$.

Tables 3 and 4 list the computational normalized stress intensity factor $f_{1}, f_{2}$, and $f_{e}$ solutions for the dissimilar aluminum/steel and magnesium/steel weld cases with $\delta=1$, respectively. For these dissimilar welds, the stress intensity factor solutions for the right cracks are also larger than those for the left crack under both pinned and clamped loading conditions. As listed in Tables 3 and 4, the bending moments and the transverse shear forces at the clamped edges reduce the stress intensity factor solutions by about 15 to $21 \%$ and 18 to $29 \%$ for the right crack, respectively, and increase the stress intensity factor solutions by about 3 to $5 \%$ and 9 to $11 \%$ for the left crack, respectively, for the dissimilar aluminum/steel and magnesium/steel weld cases. The computational results indicate that the effects of the clamped loading conditions can affect the stress intensity factor solutions much more for the dissimilar welds, especially for the magnesium/steel weld case with a larger difference in the Young's moduli.

Table 1. The computational normalized stress intensity factor $f_{I}, f_{\text {II }}$, and $f_{e}$ solutions for the similar weld in the specimen with $\delta=1$ under pinned and clamped loading conditions

\begin{tabular}{|c|c|c|c|}
\hline Computation & Pinned & Clamped & Change \\
\hline$f_{I}$ & 0.8656 & 0.7999 & $-7.59 \%$ \\
\hline$f_{I I}$ & 0.9998 & 0.9287 & $-7.11 \%$ \\
\hline$f_{e}$ & 1.3224 & 1.2257 & $-7.31 \%$ \\
\hline
\end{tabular}


Table 2. The computational normalized stress intensity factor $f_{I}, f_{\text {II }}$, and $f_{e}$ solutions for the similar weld in the specimen with $\delta=0.5$ under pinned and clamped loading conditions

\begin{tabular}{|c|c|c|c|c|c|c|}
\hline \multirow{2}{*}{ Computation } & \multicolumn{3}{|c|}{ Left } & \multicolumn{3}{c|}{ Right } \\
\cline { 2 - 7 } & Pinned & Clamped & Change & Pinned & Clamped & Change \\
\hline$f_{I}$ & 0.5903 & 0.5532 & $-6.28 \%$ & 0.8023 & 0.7533 & $-6.11 \%$ \\
\hline$f_{I I}$ & 0.6546 & 0.6157 & $-5.94 \%$ & 1.0640 & 1.0120 & $-4.89 \%$ \\
\hline$f_{e}$ & 0.8815 & 0.8277 & $-6.10 \%$ & 1.3326 & 1.2616 & $-5.33 \%$ \\
\hline
\end{tabular}

Table 3. The computational normalized stress intensity factor $f_{1}, f_{2}$, and $f_{e}$ solutions for the aluminum/steel dissimilar weld in the specimen with $\delta=1$ under pinned and clamped loading conditions

\begin{tabular}{|c|c|c|c|c|c|c|}
\hline \multirow{2}{*}{ Computation } & \multicolumn{3}{|c|}{ Left } & \multicolumn{3}{c|}{ Right } \\
\cline { 2 - 7 } & Pinned & Clamped & Change & Pinned & Clamped & Change \\
\hline$f_{1}$ & 0.6053 & 0.6356 & $+5.01 \%$ & 0.9918 & 0.7807 & $-21.28 \%$ \\
\hline$f_{2}$ & 0.6544 & 0.6732 & $+2.87 \%$ & 1.3441 & 1.1439 & $-14.89 \%$ \\
\hline$f_{e}$ & 0.8914 & 0.9258 & $+3.86 \%$ & 1.6704 & 1.3849 & $-17.09 \%$ \\
\hline
\end{tabular}

Table 4. The computational normalized stress intensity factor $f_{1}, f_{2}$, and $f_{e}$ solutions for the magnesium/steel dissimilar weld in the specimen with $\delta=1$ under pinned and clamped loading conditions

\begin{tabular}{|c|c|c|c|c|c|c|}
\hline \multirow{2}{*}{ Computation } & \multicolumn{3}{|c|}{ Left } & \multicolumn{3}{c|}{ Right } \\
\cline { 2 - 7 } & Pinned & Clamped & Change & Pinned & Clamped & Change \\
\hline$f_{1}$ & 0.5077 & 0.5652 & $+11.33 \%$ & 0.9854 & 0.6971 & $-29.26 \%$ \\
\hline$f_{2}$ & 0.5225 & 0.5668 & $+8.48 \%$ & 1.4606 & 1.1915 & $-18.42 \%$ \\
\hline$f_{e}$ & 0.7285 & 0.8004 & $+9.87 \%$ & 1.7619 & 1.3804 & $-21.65 \%$ \\
\hline
\end{tabular}




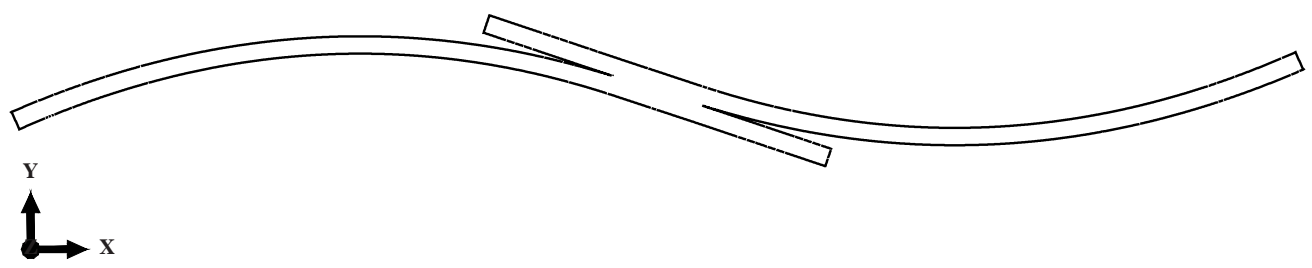

Figure 7. The deformed shape of the lap-shear specimen of similar material with $\delta=1$ under pinned loading conditions.

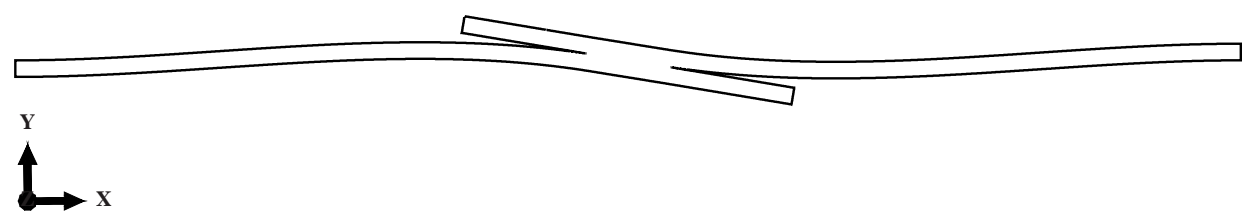

Figure 8. The deformed shape of the lap-shear specimen of similar material with $\delta=1$ under clamped loading conditions.

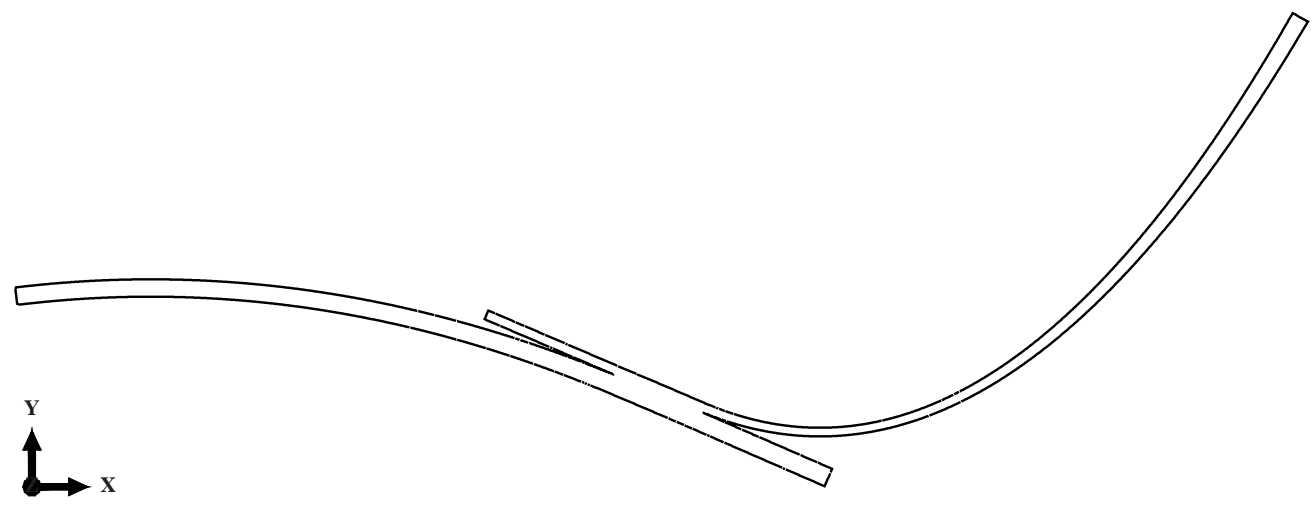

Figure 9. The deformed shape of the lap-shear specimen of similar material with $\delta=0.5$ under pinned loading conditions.

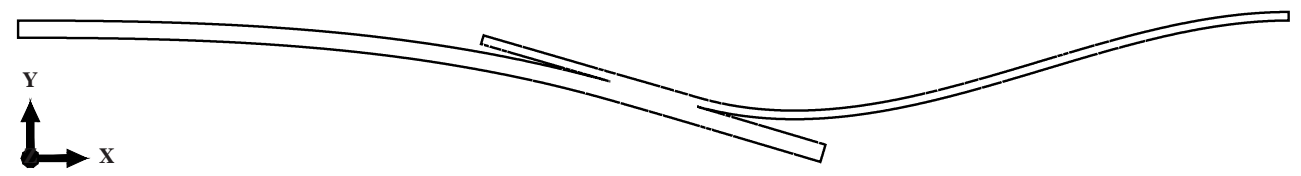

Figure 10. The deformed shape of the lap-shear specimen of similar material with $\delta=0.5$ under clamped loading conditions. 


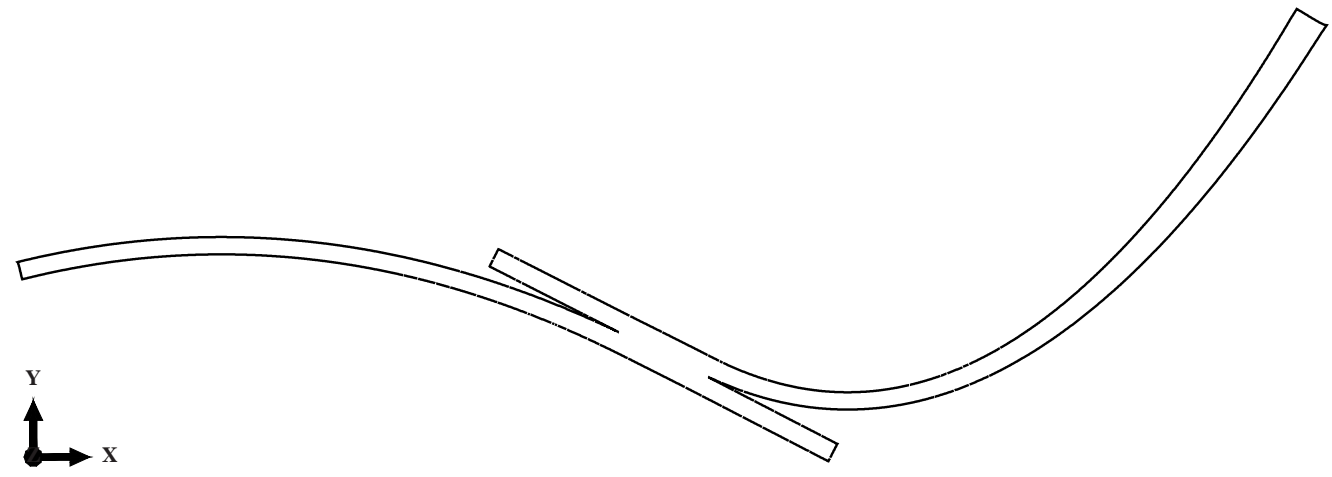

Figure 11. The deformed shape of the lap-shear specimen of dissimilar aluminum/steel with $\delta=1$ under pinned loading conditions.

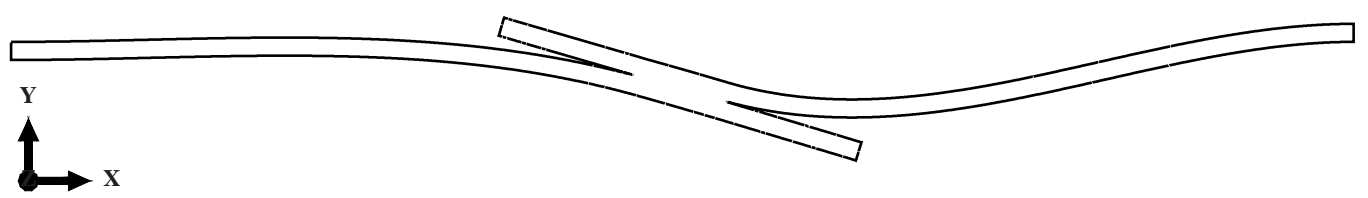

Figure 12. The deformed shape of the lap-shear specimen of dissimilar aluminum/steel with $\delta=1$ under clamped loading conditions.

The deformed shapes of the lap-shear specimens of similar material with $\delta=1$ under pinned and clamped loading conditions are shown in Figures 7 and 8, respectively. The load applied to the specimens is set at $\sqrt{t_{u}} \mathrm{~N}$ where $t_{u}$ is expressed in mm under pinned loading conditions. Under clamped loading conditions, the total load due to the applied displacement is also set at $\sqrt{t_{u}}$ N. Note that, as indicated in Equations (9) to (22) for the stress intensity factor solutions, when the load is set equal to $\sqrt{t_{u}}$ with $t_{u}$ expressed in $\mathrm{mm}$ and $b=1 \mathrm{~mm}$, the stress intensity factor solutions obtained from ABAQUS represent the normalized stress intensity factor solutions for the similar material cases and can be converted to the normalized stress intensity factor solutions for the dissimilar material cases. The scale factor is set at 1,000 to show the rotation at the pinned ends in these figures. The deformed shapes of the lap-shear specimens of 
similar material with $\delta=0.5$ under pinned and clamped loading conditions are shown in Figures 9 and 10, respectively. The deformed shapes of the lap-shear specimens of dissimilar aluminum/steel with $\delta=1$ under pinned and clamped loading conditions are shown in Figures 11 and 12, respectively. The deformed shapes of the lap-shear specimens of dissimilar magnesium/steel with $\delta=1$ under pinned and clamped loading conditions are similar to those in Figures 11 and 12, respectively, and, therefore, they are not shown here. As shown in Figures 7 to 12 , the deformations of the upper sheet of the specimens appear to be larger under pinned loading conditions. This could be used to explain the larger stress intensity factors for the right cracks in the specimens. It should be mentioned that the scale factor is set at 1,000 in Figures 7, 9 and 11 under pinned loading conditions. As shown in Figure 9 and 11, the thicknesses of the upper sheets appear to be larger toward the right edges where the rotations of the specimens are larger under pinned loading conditions.

\section{Equilibrium Equations for Lap-Shear Specimens under Different Loading Conditions}

Many different combinations of materials, thicknesses, weld lengths, and specimen lengths for the lap-shear specimens are possible. Therefore, it is useful to have general analytical solutions available under clamped loading conditions for each combination so that the effects of the clamped boundary conditions can be assessed easily from the analytical solutions. It is one of the goals of this paper that all necessary equations are provided in this paper so that one can obtain the analytical solutions from these equations for each individual application.

Figure 13(a) show a free body diagram of the lap-shear specimen subject to the force $F$ under pinned loading conditions. Figure 13(b) show a free body diagram of the lap-shear specimen under statically equivalent loading conditions. Since there is no transverse shear force 
for the lower and upper sheets, the bending moments $M_{A}$ and $M_{B}$ at A and B can be obtained as $-F t_{l} / 2$ and $F t_{u} / 2$ as shown in the figure. The moments are constant in the lower and upper sheets. Additional free body diagrams for the specimen under pinned loading conditions are shown in Figures 14(a), 14(b) and 14(c) for the lower sheet, the weld, and the upper sheet, respectively.

Figure 15 shows a free body diagram of a lap-shear specimen subject to the force $F$ under clamped loading conditions. Due to the clamped edges, the specimen edges have the transverse shear force $V$ and the bending moments $M_{A}$ and $M_{B}$ at A and B. Additional free body diagrams for the specimen are shown in Figures 16(a), 16(b) and 16(c) for the lower sheet, the weld, and the upper sheet, respectively. It should be mentioned that the upper and lower sheets are not subject to the constant bending moment conditions as in the case of the pinned loading conditions due to the transverse shear force $V$ as shown in Figures 16(a), 16(b) and 16(c). The equilibrium equation for the weld in Figure 16(b) is

$$
-M_{D}+M_{E}-2 V r-F\left(t_{u}+t_{l}\right) / 2=0
$$

Here, $M_{D}$ and $M_{E}$ are the bending moments at $\mathrm{D}$ and $\mathrm{E}$, respectively. The available beam solutions for beams $\mathrm{AD}$ and $\mathrm{BE}$ under clamped conditions give the displacements of beam $\mathrm{AD}$ and $\mathrm{BE}$ at $\mathrm{D}$ and $\mathrm{E}$ as

$$
v_{A D}=-\frac{V\left(L_{l}-r\right)^{3}}{3 E_{l}^{\prime} I_{l}}+\frac{M_{D}\left(L_{l}-r\right)^{2}}{2 E_{l}^{\prime} I_{l}}
$$

and

$$
v_{B E}=\frac{V\left(L_{u}-r\right)^{3}}{3 E_{u}^{\prime} I_{u}}+\frac{M_{E}\left(L_{u}-r\right)^{2}}{2 E_{u}^{\prime} I_{u}}
$$


Here, $I_{u}$ and $I_{l}$ represent the moments of inertia of the upper and lower sheets, respectively.

The moments of inertia are $I_{u}=b t_{u}^{3} / 12$ and $I_{l}=b t_{l}^{3} / 12$ for the rectangular cross sections of the upper and lower sheets, respectively. Here, $b$ represents the specimen width. The available beam solutions for beams $\mathrm{AD}$ and $\mathrm{BE}$ under clamped conditions give the slopes of beam $\mathrm{AD}$ and $\mathrm{BE}$ at $\mathrm{D}$ and $\mathrm{E}$ as

$$
\theta_{A D}=-\frac{V\left(L_{l}-r\right)^{2}}{2 E_{l}^{\prime} I_{l}}+\frac{M_{D}\left(L_{l}-r\right)}{E_{l}^{\prime} I_{l}}
$$

and

$$
\theta_{B E}=-\frac{V\left(L_{u}-r\right)^{2}}{2 E_{u}^{\prime} I_{u}}-\frac{M_{E}\left(L_{u}-r\right)}{E_{u}^{\prime} I_{u}}
$$

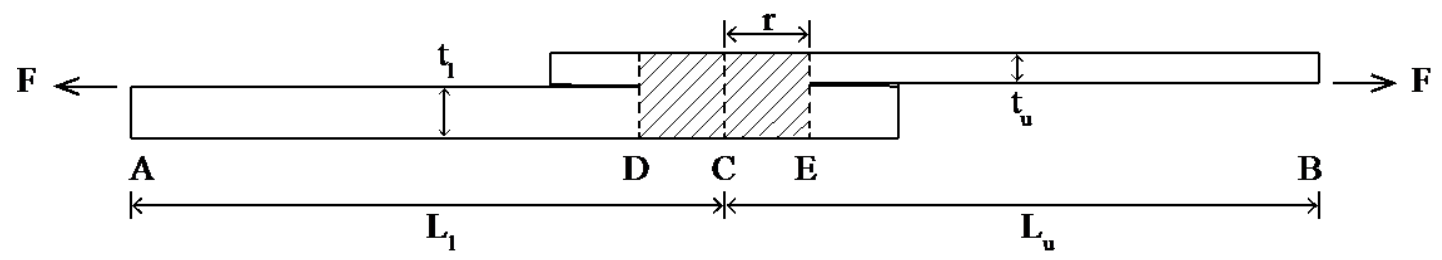

(a)

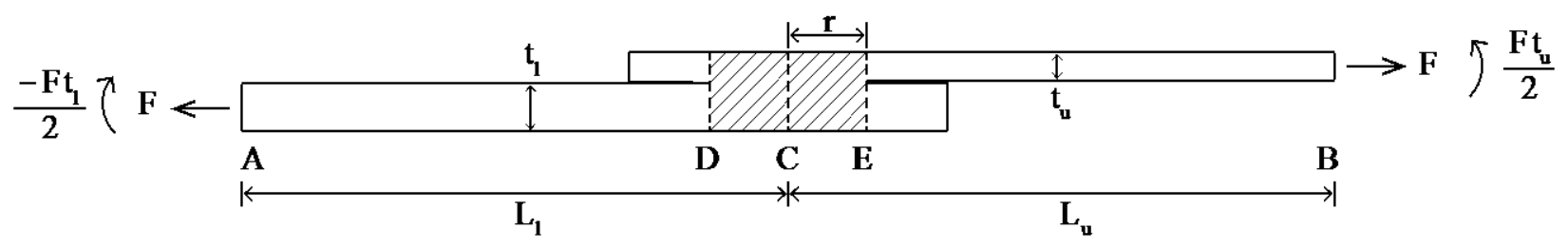

(b)

Figure 13. Free body diagrams of a lap-shear specimen (a) under pinned loading conditions and (b) under equivalent loading conditions 


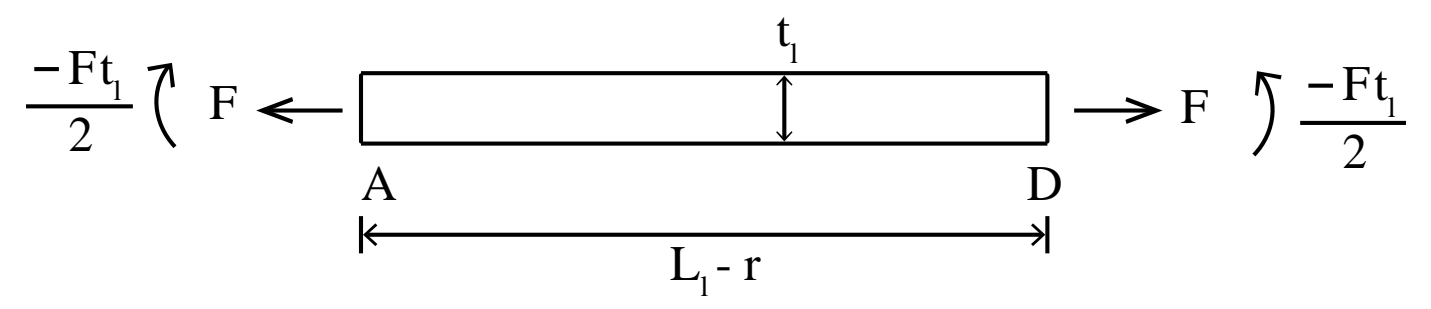

(a)

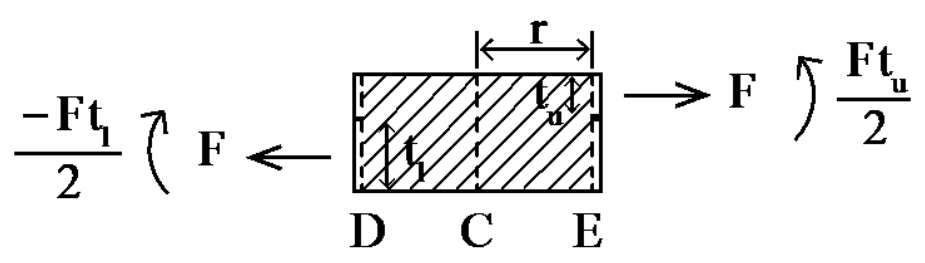

(b)

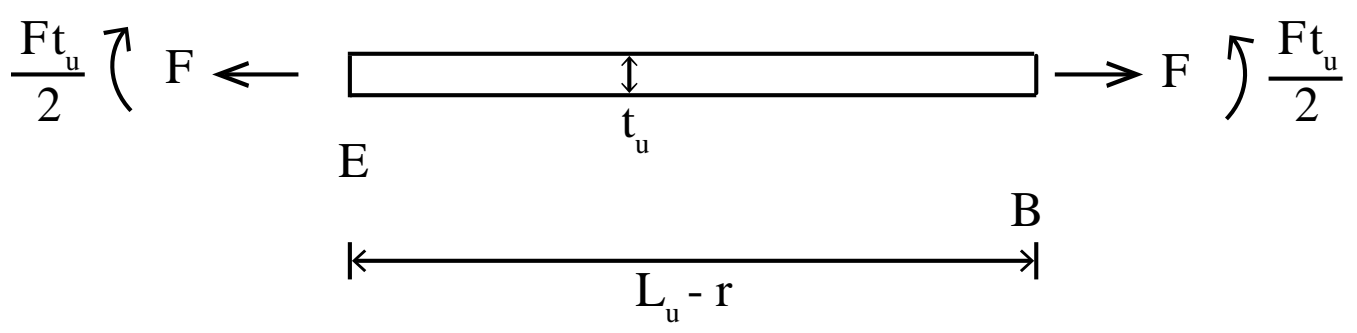

(c)

Figure 14. Free body diagrams for a lap-shear specimen under pinned loading conditions for (a) the lower sheet, (b) the weld, and (c) the upper sheet.

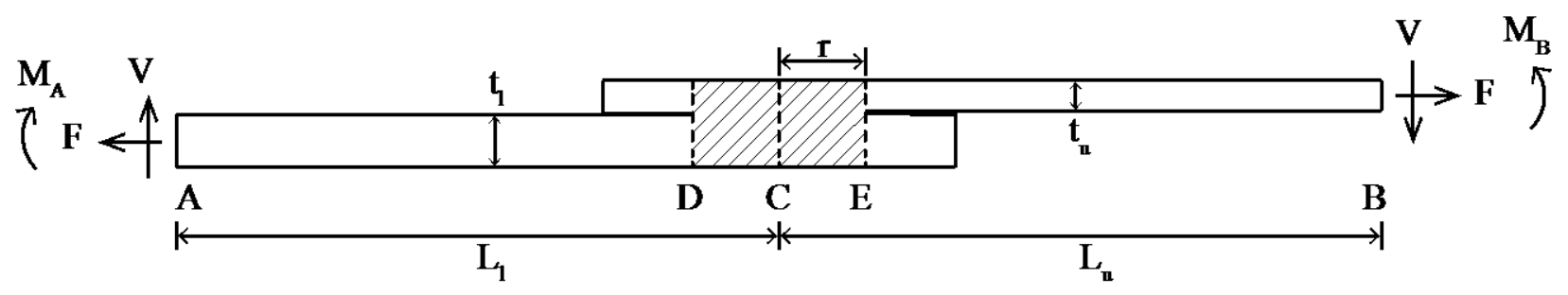

Figure 15. A free body diagram of a lap-shear specimen under clamped loading conditions. The force $F$, the bending moments $M_{A}$ and $M_{B}$, and the transverse shear force $V$ are shown at the specimen edges. 


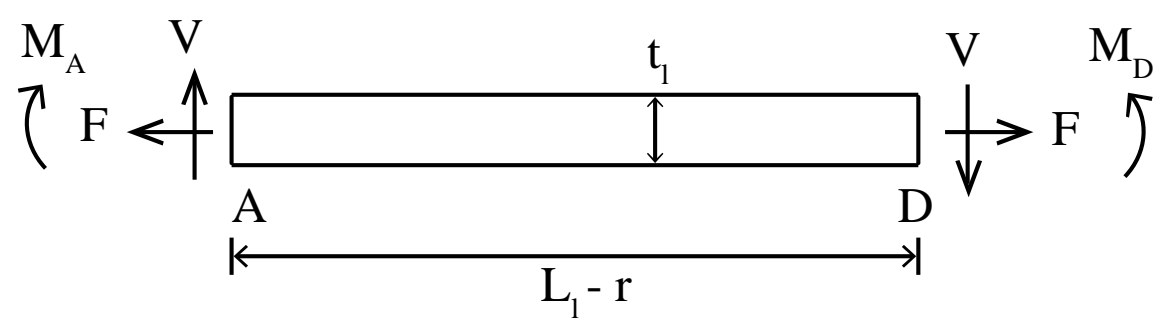

(a)

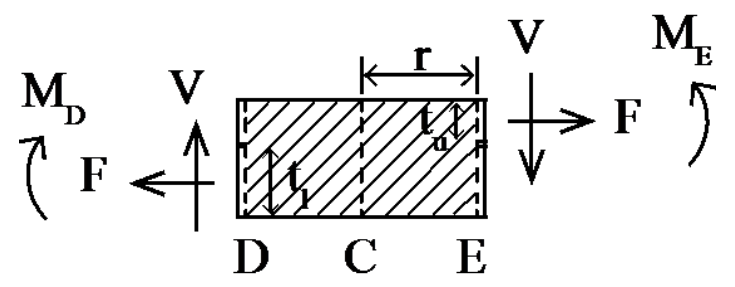

(b)

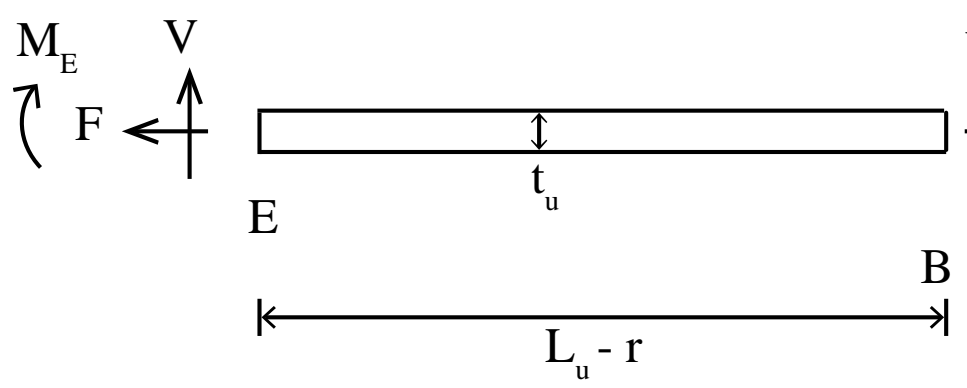

(c)

Figure 16. Free body diagrams for (a) the lower sheet, (b) the weld, and (c) the upper sheet.

In order to obtain a compact closed-form solution, the weld is assumed to be rigid, as in the mechanics analyses of spot welds in different types of specimens. When the weld DCE is treated as rigid, the displacement continuity requires that

$$
v_{A D}+2 r \theta_{A D}=v_{B E}
$$

Combining equations (24), (25), (26) and (28) gives

$$
-\frac{V\left(L_{l}-r\right)^{3}}{3 E_{l}^{\prime} I_{l}}+\frac{M_{D}\left(L_{l}-r\right)^{2}}{2 E_{l}^{\prime} I_{l}}+2 r\left(-\frac{V\left(L_{l}-r\right)^{2}}{2 E_{l}^{\prime} I_{l}}+\frac{M_{D}\left(L_{l}-r\right)}{E_{l}^{\prime} I_{l}}\right)=\frac{V\left(L_{u}-r\right)^{3}}{3 E_{u}^{\prime} I_{u}}+\frac{M_{E}\left(L_{u}-r\right)^{2}}{2 E_{u}^{\prime} I_{u}}
$$


The slope continuity for the weld at D and E gives

$$
-\frac{V\left(L_{l}-r\right)^{2}}{2 E_{l}^{\prime} I_{l}}+\frac{M_{D}\left(L_{l}-r\right)}{E_{l}^{\prime} I_{l}}=-\frac{V\left(L_{u}-r\right)^{2}}{2 E_{u}^{\prime} I_{u}}-\frac{M_{E}\left(L_{u}-r\right)}{E_{u}^{\prime} I_{u}}
$$

With Equations (23), (29) and (30), the transverse shear force $V$ and the bending moments $M_{D}$ and $M_{E}$ can be determined. In this investigation, the lengths of the upper and lower sheets are set equal to each other $\left(L_{u}=L_{l}=L\right)$. In order to list the solution for future application and compare with the solution under pinned loading conditions, the solutions for the bending moments $M_{D}$ and $M_{E}$ at D and E are expressed in the forms as listed in the following.

$$
\begin{aligned}
& M_{D}=-\frac{F t_{l}}{2}\left(1-C_{l}\left(\delta, \eta, \frac{r}{L}\right)\right) \\
& M_{E}=\frac{F t_{u}}{2}\left(1-C_{u}\left(\delta, \eta, \frac{r}{L}\right)\right)
\end{aligned}
$$

Here, $C_{l}$ and $C_{u}$ are functions of $\delta, \eta$, and $r / L$ as

$$
C_{l}=\frac{\delta\left[\left(\delta^{5} \eta^{2}-1\right)\left(1-2 \frac{r}{L}+\left(\frac{r}{L}\right)^{2}\right)+\delta^{3} \eta\left(-7+2 \frac{r}{L}+5\left(\frac{r}{L}\right)^{2}\right)+\delta^{2} \eta\left(7+22 \frac{r}{L}+19\left(\frac{r}{L}\right)^{2}\right)\right]}{\left(\delta^{6} \eta^{2}+14 \eta \delta^{3}+1\right)\left(1-2 \frac{r}{L}+\left(\frac{r}{L}\right)^{2}\right)+48 \delta^{3} \eta \frac{r}{L}}
$$

and

$$
C_{u}=\frac{-\left(\delta^{5} \eta^{2}-1\right)\left(1-2 \frac{r}{L}+\left(\frac{r}{L}\right)^{2}\right)+\delta^{2} \eta\left(-7+2 \frac{r}{L}+5\left(\frac{r}{L}\right)^{2}\right)+\delta^{3} \eta\left(7+22 \frac{r}{L}+19\left(\frac{r}{L}\right)^{2}\right)}{\left(\delta^{6} \eta^{2}+14 \eta \delta^{3}+1\right)\left(1-2 \frac{r}{L}+\left(\frac{r}{L}\right)^{2}\right)+48 \delta^{3} \eta \frac{r}{L}}
$$


The solutions of the bending moments $M_{D}$ and $M_{E}$ listed in Equations (31) to (34) are expressed in the forms so that the moments at the edges of the weld can be directly compared with those under pinned loading conditions.

The stress intensity factor solutions for elastic beams and plates can be obtained by the local membrane forces and moments or the local structural stresses as indicated in Pook [13] and Zhang [4]. Zhang [4] used the structural stresses and the $\mathrm{J}$ integral to obtain the stress intensity factor solutions. The beam at the location where the stress intensity factor solutions are evaluated are usually assumed to be long compared to the sheet thickness so that the strain energy, J integral, and the stress intensity factor solutions can be derived as in Zhang [4] and Rice [14]. For the specimen of similar material and equal thickness, the stress intensity factor solutions can be obtained by decomposition of the applied load into symmetric and antisymmetric parts so that the mode I and II stress intensity factor solutions can be obtained for each part directly. For the specimen of dissimilar material and unequal thickness, the decomposition of the stress intensity factor solutions has to go through the closed-form solutions given by Zhang [4] and Suo and Hutchinson [5]. Here, we choose to use the energy release rate approach to obtain the stress intensity factor solutions for the similar material and equal thickness case. We will then use the closed-form solutions given by Zhang [4] and Suo and Hutchinson [5] to obtain the stress intensity factor solutions for specimens of dissimilar material and unequal thickness. 


\section{Lap-Shear Specimens of Similar Material and Equal Thickness}

Here, the upper and lower sheets are the similar material and the thicknesses are set equal to each other $\left(t_{u}=t_{l}=t\right)$. Under pinned loading conditions, the transverse shear force $V$ and the bending moments $M_{D}$ and $M_{E}$ as shown in Figure 14(b) are expressed as

$$
\begin{aligned}
& V=0 \\
& M_{D}=-\frac{1}{2} F t \\
& M_{E}=\frac{1}{2} F t
\end{aligned}
$$

Under clamped loading conditions, the solutions for the transverse shear force $V$ and the bending moments $M_{D}$ and $M_{E}$ at $\mathrm{D}$ and $\mathrm{E}$ can be obtained from Equations (31) to (34) by setting up $\delta=1$ and $\eta=1$. For this case, $C_{u}=C_{l}=C$. Here, $C$ is expressed as

$$
C=\frac{3}{2} \frac{r}{L}\left(\frac{1+\frac{r}{L}}{1+\frac{r}{L}+\left(\frac{r}{L}\right)^{2}}\right)
$$

The solutions for the transverse force $V$ and the bending moments $M_{D}$ and $M_{E}$ can be expressed in the compact forms as

$$
\begin{aligned}
& V=-\frac{3}{4} F \frac{t}{L} \lambda \\
& M_{D}=-\frac{1}{2} F t\left(1-\frac{3}{2} \frac{r}{L} \lambda\right) \\
& M_{E}=\frac{1}{2} F t\left(1-\frac{3}{2} \frac{r}{L} \lambda\right)
\end{aligned}
$$

where 


$$
\lambda=\frac{1+\frac{r}{L}}{1+\frac{r}{L}+\left(\frac{r}{L}\right)^{2}}
$$

Here, $\lambda$ is a function of $r / L$ as in Equation (42). As indicated in Equations (39) to (42), the bending moments $M_{D}$ and $M_{E}$ depend on the ratio $r / L$, and the transverse shear force $V$ depends on the ratios $t / L$ and $r / L$ under clamped loading conditions. When $r$ approaches to 0, $\lambda$ approaches to 1 and the bending moment solutions used in Hong et al. [7] and Sung and Pan [8] are recovered.

Figure 17 shows a schematic of the decomposition of the load at $\mathrm{E}$ based on the beam theory. As indicated in Zhang [4], the transverse shear force contribution is small and therefore neglected in this investigation. Figure 17(a) shows the beam model under the load at E. The original load in Figure 17(a) is then decomposed into the loads that give the (b) counter bending, (c) central bending, (d) tension, and (e) in-plane shear loading conditions, without consideration of the transverse shear force. Based on the beam theory, when the crack grows by a small increment $d x$, the energy release rate can be obtained for cases (b) to (e) as in Appendix A. The counter bending case gives the mode I stress intensity factor. The central bending and in-plane shear loading cases give the mode II stress intensity factors. The tension case induces no stress intensity factor. 


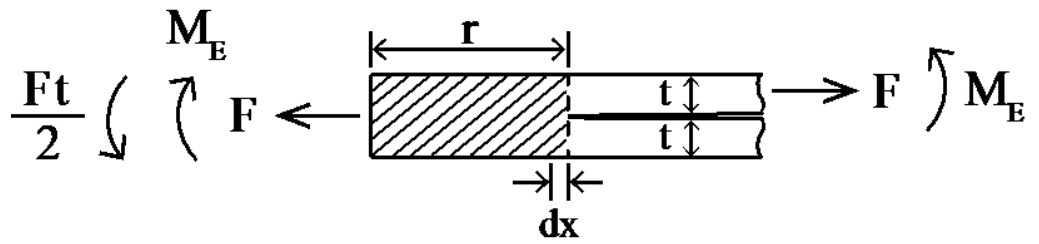

(a)

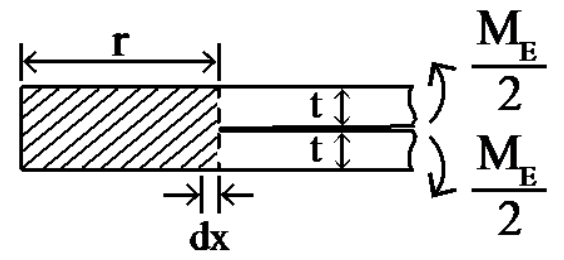

(b)

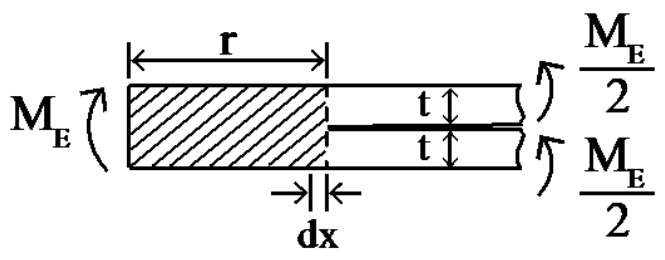

(c)

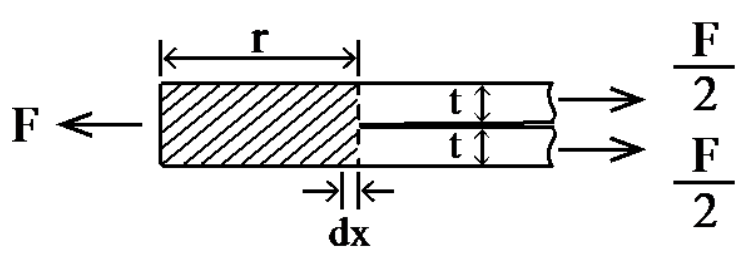

(d)

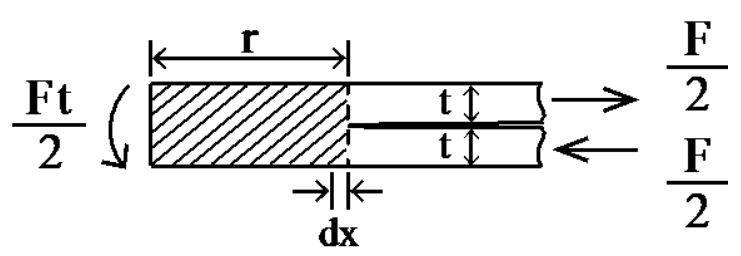

(e)

Figure 17. A schematic of the decomposition of the load at E based on the beam theory. (a) The load at E. The original load in (a) is decomposed into the loads that give the (b) counter bending, (c) central bending, (d) tension, and (e) in-plane shear loading conditions without consideration of the transverse shear force. 
Under pinned loading conditions, the stress intensity factor solutions for the equal thickness case in the normalized forms were obtained in Sripichai et al [1] as

$$
\begin{aligned}
& K_{I}=f_{I}^{p} \frac{F}{b \sqrt{t}} \\
& K_{I I}=f_{I I}^{p} \frac{F}{b \sqrt{t}}
\end{aligned}
$$

where $f_{I}^{p}$ and $f_{I I}^{p}$ are

$$
\begin{aligned}
& f_{I}^{p}=\frac{\sqrt{3}}{2} \\
& f_{I I}^{p}=1
\end{aligned}
$$

The effective stress intensity factor $K_{e}$ is defined in Equation (18) and the normalized stress intensity factor $f_{e}^{p}$ can be obtained easily.

Under clamped loading conditions, the stress intensity factor solutions for the equal thickness case in the normalized forms can be expressed as

$$
\begin{aligned}
& K_{I}=f_{I}^{c}\left(\frac{r}{L}\right) \frac{F}{b \sqrt{t}} \\
& K_{I I}=f_{I I}^{c}\left(\frac{r}{L}\right) \frac{F}{b \sqrt{t}}
\end{aligned}
$$

where $f_{I}^{c}$ and $f_{I I}^{c}$ are the dimensionless geometric functions of $r / L$ as

$$
\begin{aligned}
& f_{I}^{c}=\frac{\sqrt{3}}{2}-\frac{3 \sqrt{3}}{4} \frac{r}{L} \lambda \\
& f_{I I}^{c}=1-\frac{9}{8} \frac{r}{L} \lambda
\end{aligned}
$$


The effective stress intensity factor $K_{e}$ is defined in Equation (18) and the normalized stress intensity factor $f_{e}^{c}$ can also be obtained easily.

\section{Lap-Shear Specimens of Dissimilar Material and Unequal Thickness}

For lap-shear specimens of dissimilar material and unequal thickness, the solutions for the bending moments $M_{D}$ and $M_{E}$ are listed in Equations (31) to (34). It is difficult to decompose the loading condition into the parts where $K_{1}, K_{2}, K_{I}$ and $K_{I I}$ can be extracted directly.

Therefore, the decomposition to $K_{1}, K_{2}, K_{I}$ and $K_{I I}$ of Zhang [4] is used here. The local beam model of Zhang [4] is shown in Figure 18. Here, the normal stresses $\sigma_{u i}, \sigma_{u o}, \sigma_{l i}, \sigma_{l o}, \sigma_{u i}^{*}$, $\sigma_{u o}^{*}, \sigma_{l i}^{*}$ and $\sigma_{l o}^{*}$ represent the normal structural stresses at the inner (i) and outer (o) surfaces of the upper (u) and lower (l) sheets, respectively. The normal stresses $\sigma_{u i}^{*}, \sigma_{u o}^{*}, \sigma_{l i}^{*}$ and $\sigma_{l o}^{*}$ can be expressed in terms of the normal stresses $\sigma_{u i}, \sigma_{u o}, \sigma_{l i}$, and $\sigma_{l o}$. The $K_{1}$ and $K_{2}$ solutions from Zhang [4] can be expressed in terms of $\sigma_{u i}, \sigma_{u o}, \sigma_{l i}$, and $\sigma_{l o}$ as

$$
\begin{aligned}
& K_{1}=\frac{\cosh (\pi \varepsilon) \sqrt{t_{u}}}{2 \sqrt{3(1+\eta)\left(1+4 \eta \delta+6 \eta \delta^{2}+3 \eta \delta^{3}\right)\left(1+\tan ^{2} \omega\right)}} \times \\
& \left\{\left[\frac{\left(1+4 \eta \delta+9 \eta \delta^{2}+6 \eta \delta^{3}\right) \tan \omega}{\sqrt{1+2 \eta \delta\left(2+3 \delta+2 \delta^{2}\right)+\eta^{2} \delta^{4}}}-\sqrt{3}\right] \sigma_{u i}\right. \\
& -\left[\frac{\left(1+4 \eta \delta+3 \eta \delta^{2}\right) \tan \omega}{\sqrt{1+2 \eta \delta\left(2+3 \delta+2 \delta^{2}\right)+\eta^{2} \delta^{4}}}+\sqrt{3}\right] \sigma_{u o} \\
& +\eta\left[\frac{\delta\left(1-2 \eta \delta-3 \eta \delta^{2}\right) \tan \omega}{\sqrt{1+2 \eta \delta\left(2+3 \delta+2 \delta^{2}\right)+\eta^{2} \delta^{4}}}+\sqrt{3}(2+\delta)\right] \sigma_{l i} \\
& \left.-\eta \delta\left[\frac{\left(1+4 \eta \delta+3 \eta \delta^{2}\right) \tan \omega}{\sqrt{1+2 \eta \delta\left(2+3 \delta+2 \delta^{2}\right)+\eta^{2} \delta^{4}}}+\sqrt{3}\right] \sigma_{l o}\right\}
\end{aligned}
$$




$$
\begin{aligned}
& K_{2}=\frac{\cosh (\pi \varepsilon) \sqrt{t_{u}}}{2 \sqrt{3(1+\eta)\left(1+4 \eta \delta+6 \eta \delta^{2}+3 \eta \delta^{3}\right)\left(1+\tan ^{2} \omega\right)}} \times \\
& \left\{\left[\frac{1+4 \eta \delta+9 \eta \delta^{2}+6 \eta \delta^{3}}{\sqrt{1+2 \eta \delta\left(2+3 \delta+2 \delta^{2}\right)+\eta^{2} \delta^{4}}}+\sqrt{3} \tan \omega\right] \sigma_{u i}\right. \\
& -\left[\frac{1+4 \eta \delta+3 \eta \delta^{2}}{\sqrt{1+2 \eta \delta\left(2+3 \delta+2 \delta^{2}\right)+\eta^{2} \delta^{4}}}-\sqrt{3} \tan \omega\right] \sigma_{u o} \\
& +\eta\left[\frac{\delta\left(1-2 \eta \delta-3 \eta \delta^{2}\right)}{\sqrt{1+2 \eta \delta\left(2+3 \delta+2 \delta^{2}\right)+\eta^{2} \delta^{4}}}-\sqrt{3}(2+\delta) \tan \omega\right] \sigma_{l i} \\
& \left.-\eta \delta\left[\frac{1+4 \eta \delta+3 \eta \delta^{2}}{\sqrt{1+2 \eta \delta\left(2+3 \delta+2 \delta^{2}\right)+\eta^{2} \delta^{4}}}-\sqrt{3} \tan \omega\right] \sigma_{l o}\right\}
\end{aligned}
$$

Note that the expressions for the $K_{1}$ and $K_{2}$ solutions are for $\delta \leq 1$. The values of the angular quantity $\omega$ in Equations (51) and (52) can be found in Suo and Hutchinson [5] and Zhang [4]. For the similar material $(\eta=1)$, the $K_{1}$ and $K_{2}$ solutions become $K_{I}$ and $K_{I I}$ which are listed in the following.

$$
\begin{aligned}
& K_{I}=\frac{\sqrt{t_{u}}}{2 \sqrt{2(1+\delta)\left(1+3 \delta+3 \delta^{2}\right)\left(1+\tan ^{2} \omega\right)}} \times \\
& \left\{\left[\frac{1+3 \delta+6 \delta^{2}}{\sqrt{3}(1+\delta)} \tan \omega-1\right] \sigma_{u i}-\left[\frac{1+3 \delta}{\sqrt{3}(1+\delta)} \tan \omega+1\right] \sigma_{u o}\right. \\
& \left.+\left[\frac{\delta(1-3 \delta)}{\sqrt{3}(1+\delta)} \tan \omega+2+\delta\right] \sigma_{l i}-\delta\left[\frac{1+3 \delta}{\sqrt{3}(1+\delta)} \tan \omega+1\right] \sigma_{l o}\right\} \\
& K_{I I}=\frac{\sqrt{t_{u}}}{2 \sqrt{2(1+\delta)\left(1+3 \delta+3 \delta^{2}\right)\left(1+\tan ^{2} \omega\right)}} \times \\
& \left\{\left[\frac{1+3 \delta+6 \delta^{2}}{\sqrt{3}(1+\delta)}+\tan \omega\right] \sigma_{u i}-\left[\frac{1+3 \delta}{\sqrt{3}(1+\delta)}-\tan \omega\right] \sigma_{u o}\right. \\
& \left.+\left[\frac{\delta(1-3 \delta)}{\sqrt{3}(1+\delta)}-(2+\delta) \tan \omega\right] \sigma_{l i}-\delta\left[\frac{1+3 \delta}{\sqrt{3}(1+\delta)}-\tan \omega\right] \sigma_{l o}\right\}
\end{aligned}
$$




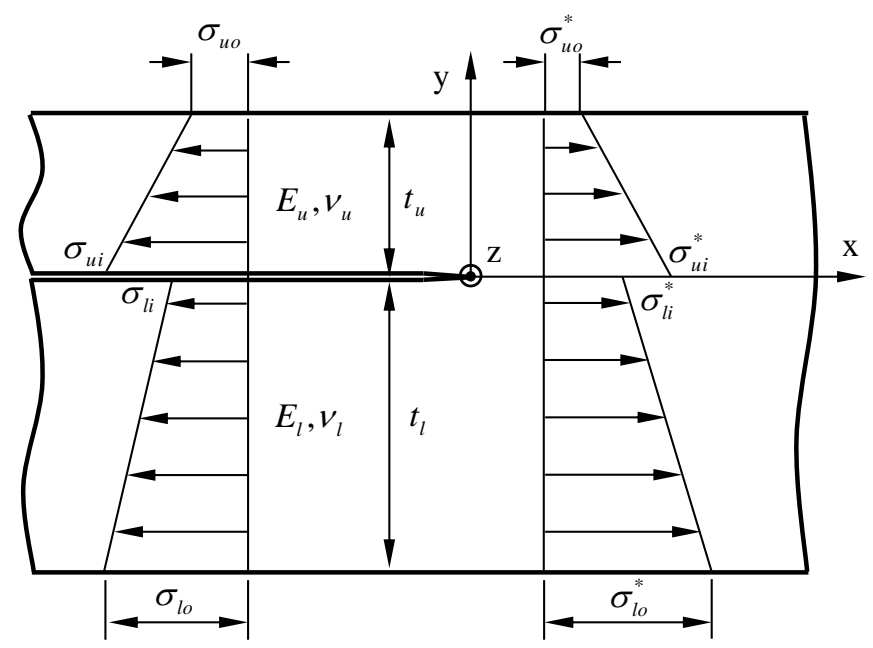

Figure 18. A schematic of the beam model near the crack tip. The normal stresses $\sigma_{u i}, \sigma_{u o}, \sigma_{l i}$, $\sigma_{l o}, \sigma_{u i}^{*}, \sigma_{u o}^{*}, \sigma_{l i}^{*}$ and $\sigma_{l o}^{*}$ represent the normal structural stresses at the inner (i) and outer (o) surfaces of the upper (u) and lower (1) strips, respectively.

The normal structural stresses for the left and right cracks in a lap-shear specimen can be obtained from the applied force $F$ and the bending moments $M_{D}$ and $M_{E}$ using the beam theory. For the left crack, the normal structural stresses are

$$
\begin{aligned}
& \sigma_{u o}=0 \\
& \sigma_{u i}=0 \\
& \sigma_{l i}=\frac{F}{b t_{l}}-\frac{6 M_{D}}{b t_{l}^{2}} \\
& \sigma_{l o}=\frac{F}{b t_{l}}+\frac{6 M_{D}}{b t_{l}^{2}}
\end{aligned}
$$

For the right crack, the normal structural stresses are

$$
\sigma_{u o}=\frac{F}{b t_{u}}-\frac{6 M_{E}}{b t_{u}^{2}}
$$




$$
\begin{aligned}
& \sigma_{u i}=\frac{F}{b t_{u}}+\frac{6 M_{E}}{b t_{u}^{2}} \\
& \sigma_{l i}=0 \\
& \sigma_{l o}=0
\end{aligned}
$$

For specimens of dissimilar material under pinned loading conditions, the stress intensity factor $K_{1}, K_{2}$, and $K_{e}$ solutions can be expressed in the normalized forms as

$$
\begin{aligned}
& K_{1}=f_{1}^{p}(\eta, \delta) \frac{F}{b \sqrt{t_{u}}} \\
& K_{2}=f_{2}^{p}(\eta, \delta) \frac{F}{b \sqrt{t_{u}}} \\
& K_{e}=f_{e}^{p}(\eta, \delta) \frac{F}{b \sqrt{t_{u}}}
\end{aligned}
$$

where the superscript " $\mathrm{p}$ " represents the pinned loading conditions. Here, $f_{1}^{p}, f_{2}^{p}$, and $f_{e}^{p}$ are the normalized stress intensity factor solutions for the pinned loading conditions. The stress intensity factor $K_{1}, K_{2}$ and $K_{e}$ solutions for a weld in a lap-shear specimen under clamped loading conditions can also be expressed in the normalized forms as

$$
\begin{aligned}
& K_{1}=f_{1}^{c}\left(\eta, \delta, \frac{r}{L}\right) \frac{F}{b \sqrt{t_{u}}} \\
& K_{2}=f_{2}^{c}\left(\eta, \delta, \frac{r}{L}\right) \frac{F}{b \sqrt{t_{u}}} \\
& K_{e}=f_{e}^{c}\left(\eta, \delta, \frac{r}{L}\right) \frac{F}{b \sqrt{t_{u}}}
\end{aligned}
$$


where the superscript " $\mathrm{c}$ " represents the clamped loading conditions. Here, $f_{1}^{c}, f_{2}^{c}$, and $f_{e}^{c}$ are the normalized stress intensity factor solutions for the clamped loading conditions.

For specimens of similar material under pinned loading conditions, the $K_{I}, K_{I I}$ and $K_{e}$ solutions can be expressed in the normalized forms as

$$
\begin{aligned}
& K_{I}=f_{I}^{p}(\delta) \frac{F}{b \sqrt{t_{u}}} \\
& K_{I I}=f_{I I}^{p}(\delta) \frac{F}{b \sqrt{t_{u}}} \\
& K_{e}=f_{e}^{p}(\delta) \frac{F}{b \sqrt{t_{u}}}
\end{aligned}
$$

For specimens of similar material under clamped loading conditions, the $K_{I}, K_{I I}$ and $K_{e}$ solutions can be expressed in the normalized forms as

$$
\begin{gathered}
K_{I}=f_{I}^{c}\left(\delta, \frac{r}{L}\right) \frac{F}{b \sqrt{t_{u}}} \\
K_{I I}=f_{I I}^{c}\left(\delta, \frac{r}{L}\right) \frac{F}{b \sqrt{t_{u}}} \\
K_{e}=f_{e}^{c}\left(\delta, \frac{r}{L}\right) \frac{F}{b \sqrt{t_{u}}}
\end{gathered}
$$

The $K_{I}, K_{I I}$ and $K_{e}$ solutions are quite long based on the solutions for the bending moments $M_{D}$ and $M_{E}$ under clamped loading conditions and only the numerical solutions for the case of $\delta=0.5$ will be presented. When $t_{u}=t_{l}=t, \delta=1$. For the $\delta=1$ case, $\omega=\cos ^{-1}(\sqrt{3 / 7})$. The $K_{I}$ and $K_{I I}$ solutions can be simplified as 


$$
\begin{aligned}
& K_{I}=\frac{1}{6}\left(\frac{\sqrt{3}}{2}\left(\sigma_{u i}-\sigma_{u o}+\sigma_{l i}-\sigma_{l o}\right)\right) \sqrt{t} \\
& K_{I I}=\left(\frac{1}{4}\left(\sigma_{u i}-\sigma_{l i}\right)\right) \sqrt{t}
\end{aligned}
$$

When the solutions for the bending moments $M_{D}$ and $M_{E}$ are substituted into the structural stress solutions in Equations (55) to (62) and then into the $K_{I}$ and $K_{I I}$ solutions in Equations (75) and (76), the closed-form solutions in Equations (47) to (50) can be obtained. However, the closed form solutions in Equations (47) to (50) based on the energy release rate approach as shown in Appendix A can be obtained without using the solutions of Zhang [4] and Suo and Hutchinson [5].

\section{Comparison of Computational and Analytical Solutions}

Here, the computational solutions for the two similar weld cases as considered earlier and the analytical solutions based on the beam theory are compared. Tables 5 and 6 list the analytical and computational normalized stress intensity factor $f_{I}^{p}, f_{I I}^{p}$, and $f_{e}^{p}$ solutions for the similar welds in the specimens with $\delta=1$ and $\delta=0.5$, respectively, under the pinned loading conditions. As listed in the tables, the computational solutions agree very well with the analytical solutions for $\delta=1$. It should be mentioned that the cracks are under dominant mode II loading conditions and the values of the normalized mode II stress intensity factor $f_{I I}^{p}$ solutions are more important for the calculation of the normalized effective stress intensity factor $f_{e}^{p}$ solutions. For $\delta=0.5$, the computational normalized model II stress intensity factors $f_{I I}^{p}$ solutions have the error less than $2 \%$. The effective computational normalized stress intensity 
factor $f_{e}^{p}$ solutions related to the energy release rate or the $\mathrm{J}$ integral agree very well with the analytical solutions. The right crack has larger stress intensity factor solutions since the upper sheet is thinner. The computational results presented in Tables 5 and 6 are in agreement with the results presented in Lai and Pan [6].

Tables 7 and 8 list the analytical and computational normalized stress intensity factor $f_{I}^{c}$, $f_{I I}^{c}$ and $f_{e}^{c}$ solutions for the similar welds in the specimens with $\delta=1$ and $\delta=0.5$, respectively, under clamped loading conditions. Again, the cracks are under dominant mode II loading condition and the values of $f_{I I}^{c}$ are more important for the calculation of the normalized effective stress intensity factor $f_{e}^{c}$ solutions. As listed in the table, the analytical $f_{I I}^{c}$ and $f_{e}^{c}$ solutions agree reasonably well with the computational solutions with errors of less than about $2 \%$.

Table 5. The normalized stress intensity factor $f_{I}^{p}, f_{I I}^{p}$, and $f_{e}^{p}$ solutions for the similar weld in the specimen with $\delta=1$ under pinned loading conditions

\begin{tabular}{|c|c|c|c|}
\hline Pinned & Analytical & Computational & Error \\
\hline$f_{I}^{p}$ & 0.8660 & 0.8656 & $-0.05 \%$ \\
\hline$f_{I I}^{p}$ & 1.0000 & 0.9998 & $-0.02 \%$ \\
\hline$f_{e}^{p}$ & 1.3229 & 1.3224 & $-0.04 \%$ \\
\hline
\end{tabular}

Table 6. The normalized stress intensity factor $f_{I}^{p}, f_{I I}^{p}$, and $f_{e}^{p}$ solutions for the similar weld in the specimen with $\delta=0.5$ under pinned loading conditions

\begin{tabular}{|c|c|c|c|c|c|c|}
\hline \multirow{2}{*}{ Pinned } & \multicolumn{3}{|c|}{ Left } & \multicolumn{3}{c|}{ Right } \\
\cline { 2 - 7 } & Analytical & Computational & Error & Analytical & Computational & Error \\
\hline$f_{I}^{p}$ & 0.6037 & 0.5903 & $-2.22 \%$ & 0.7818 & 0.8023 & $2.62 \%$ \\
\hline$f_{I I}^{p}$ & 0.6428 & 0.6546 & $1.84 \%$ & 1.0800 & 1.0640 & $-1.48 \%$ \\
\hline$f_{e}^{p}$ & 0.8818 & 0.8815 & $-0.03 \%$ & 1.3333 & 1.3326 & $-0.05 \%$ \\
\hline
\end{tabular}


Table 7. The normalized stress intensity factor $f_{I}^{c}, f_{I I}^{c}$, and $f_{e}^{c}$ solutions for the similar weld in the specimen with $\delta=1$ under clamped loading conditions

\begin{tabular}{|c|c|c|c|}
\hline Clamped & Analytical & Computational & Error \\
\hline$f_{I}^{c}$ & 0.7767 & 0.7999 & $2.99 \%$ \\
\hline$f_{I I}^{c}$ & 0.9226 & 0.9287 & $0.66 \%$ \\
\hline$f_{e}^{c}$ & 1.2060 & 1.2257 & $1.63 \%$ \\
\hline
\end{tabular}

Table 8. The normalized stress intensity factor $f_{I}^{p}, f_{I I}^{p}$, and $f_{e}^{p}$ solutions for the similar weld in the specimen with $\delta=0.5$ under clamped loading conditions

\begin{tabular}{|c|c|c|c|c|c|c|}
\hline \multirow{2}{*}{ Clamped } & \multicolumn{3}{|c|}{ Left } & \multicolumn{3}{c|}{ Right } \\
\cline { 2 - 7 } & Analytical & Computational & Error & Analytical & Computational & Error \\
\hline$f_{I}^{c}$ & 0.5601 & 0.5532 & $-1.23 \%$ & 0.7145 & 0.7533 & $5.43 \%$ \\
\hline$f_{I I}^{c}$ & 0.6028 & 0.6157 & $2.14 \%$ & 1.0231 & 1.0120 & $-1.08 \%$ \\
\hline$f_{e}^{c}$ & 0.8228 & 0.8277 & $0.60 \%$ & 1.2479 & 1.2616 & $1.10 \%$ \\
\hline
\end{tabular}

Table 9. The analytical normalized stress intensity factor $f_{I}, f_{I I}$, and $f_{e}$ solutions for the similar weld in the specimen with $\delta=1$ under pinned and clamped loading conditions

\begin{tabular}{|c|c|c|c|}
\hline Analytical & Pinned & Clamped & Change \\
\hline$f_{I}$ & 0.8660 & 0.7767 & $-10.31 \%$ \\
\hline$f_{I I}$ & 1.0000 & 0.9226 & $-7.74 \%$ \\
\hline$f_{e}$ & 1.3229 & 1.2060 & $-8.84 \%$ \\
\hline
\end{tabular}

Table 10. The analytical normalized stress intensity factor $f_{I}, f_{I I}$, and $f_{e}$ solutions for the similar weld in the specimen with $\delta=0.5$ under pinned and clamped loading conditions

\begin{tabular}{|c|c|c|c|c|c|c|}
\hline \multirow{2}{*}{ Analytical } & \multicolumn{3}{|c|}{ Left } & \multicolumn{3}{c|}{ Right } \\
\cline { 2 - 7 } & Pinned & Clamped & Change & Pinned & Clamped & Change \\
\hline$f_{I}$ & 0.6037 & 0.5601 & $-7.22 \%$ & 0.7818 & 0.7145 & $-8.61 \%$ \\
\hline$f_{I I}$ & 0.6428 & 0.6028 & $-6.22 \%$ & 1.0800 & 1.0231 & $-5.27 \%$ \\
\hline$f_{e}$ & 0.8818 & 0.8228 & $-6.69 \%$ & 1.3333 & 1.2479 & $-6.41 \%$ \\
\hline
\end{tabular}


The analytical solutions for the similar welds appear to be quite accurate, especially for the pinned loading conditions. The analytical solutions have errors less than about $2 \%$. Tables 9 and 10 list the analytical normalized stress intensity factor $f_{I}, f_{I I}$, and $f_{e}$ solutions for the similar welds in the specimens with $\delta=1$ and $\delta=0.5$, respectively, under pinned and clamped loading conditions. As listed in the tables, the bending moments and the transverse shear forces at the clamped edges reduce the stress intensity factor solutions by about 5 to $10 \%$ for the cases of $\delta=1$ and $\delta=0.5$ for the given ratio of $r / L=0.069$. It appears that the effects of the clamped loading conditions for the similar welds can be obtained from the analytical solutions.

Table 11. The normalized stress intensity factor $f_{1}^{p}, f_{2}^{p}$, and $f_{e}^{p}$ solutions for the dissimilar aluminum/steel weld in the specimen with $\delta=1$ under pinned loading conditions

\begin{tabular}{|c|c|c|c|c|c|c|}
\hline \multirow{2}{*}{ Pinned } & \multicolumn{3}{|c|}{ Left } & \multicolumn{3}{c|}{ Right } \\
\cline { 2 - 7 } & Analytical & Computational & Error & Analytical & Computational & Error \\
\hline$f_{1}^{p}$ & 0.6041 & 0.6053 & $0.20 \%$ & 1.0351 & 0.9918 & $-4.18 \%$ \\
\hline$f_{2}^{p}$ & 0.6559 & 0.6544 & $-0.23 \%$ & 1.3112 & 1.3441 & $2.51 \%$ \\
\hline$f_{e}^{p}$ & 0.8917 & 0.8914 & $-0.03 \%$ & 1.6705 & 1.6704 & $-0.01 \%$ \\
\hline
\end{tabular}

Table 12. The normalized stress intensity factor $f_{1}^{c}, f_{2}^{c}$, and $f_{e}^{c}$ solutions for the dissimilar aluminum/steel weld in the specimen with $\delta=1$ under clamped loading conditions

\begin{tabular}{|c|c|c|c|c|c|c|}
\hline \multirow{2}{*}{ Clamped } & \multicolumn{3}{|c|}{ Left } & \multicolumn{3}{c|}{ Right } \\
\cline { 2 - 7 } & Analytical & Computational & Error & Analytical & Computational & Error \\
\hline$f_{1}^{p}$ & 0.6191 & 0.6356 & $2.67 \%$ & 0.7871 & 0.7807 & $-0.81 \%$ \\
\hline$f_{2}^{p}$ & 0.6695 & 0.6732 & $0.55 \%$ & 1.1118 & 1.1439 & $2.89 \%$ \\
\hline$f_{e}^{p}$ & 0.9119 & 0.9258 & $1.52 \%$ & 1.3622 & 1.3849 & $1.67 \%$ \\
\hline
\end{tabular}


Table 13. The analytical normalized stress intensity factor $f_{1}, f_{2}$, and $f_{e}$ solutions for the dissimilar aluminum/steel weld in the specimen with $\delta=1$ under pinned and clamped loading conditions

\begin{tabular}{|c|c|c|c|c|c|c|}
\hline \multirow{2}{*}{ Analytical } & \multicolumn{3}{|c|}{ Left } & \multicolumn{3}{c|}{ Right } \\
\cline { 2 - 7 } & Pinned & Clamped & Change & Pinned & Clamped & Change \\
\hline$f_{1}$ & 0.6041 & 0.6191 & $+2.48 \%$ & 1.0351 & 0.7871 & $-23.96 \%$ \\
\hline$f_{2}$ & 0.6559 & 0.6695 & $+2.07 \%$ & 1.3112 & 1.1118 & $-15.21 \%$ \\
\hline$f_{e}$ & 0.8917 & 0.9119 & $+2.27 \%$ & 1.6705 & 1.3622 & $-18.46 \%$ \\
\hline
\end{tabular}

Table 11 lists the analytical and computational normalized stress intensity factor $f_{1}^{p}, f_{2}^{p}$, and $f_{e}^{p}$ solutions for the dissimilar aluminum/steel weld in the specimen with $\delta=1$ under pinned loading conditions. As listed in the table, the computational solutions agree well with the analytical solutions for $\delta=1$. It should be mentioned that the cracks are under dominant mode II loading conditions and the values of the normalized stress intensity factor $f_{2}^{p}$ solution are more important for the calculation of the normalized effective stress intensity factor $f_{e}^{p}$ solutions. The right crack has larger stress intensity factor solutions since the upper sheet has a smaller elastic modulus. For the right crack, the stress intensity factors $f_{2}^{p}$ solution has the error less than $3 \%$. However, the effective computational normalized stress intensity factor $f_{e}^{p}$ solution agrees very well with the analytical solution. The computational results presented in Table 11 are in agreement with the results presented in Lai and Pan [6].

Table 12 lists the analytical and computational normalized stress intensity factor $f_{1}^{c}, f_{2}^{c}$, and $f_{e}^{c}$ solutions for the dissimilar aluminum/steel weld in the specimen with $\delta=1$ under clamped loading conditions. Again, the cracks are under dominant mode II loading condition and the values of $f_{2}^{c}$ are more important for the calculation of the normalized effective stress 
intensity factor $f_{e}^{c}$ solutions. As listed in the table, the analytical stress intensity factor $f_{2}^{c}$ and $f_{e}^{c}$ solutions agree reasonably well with the computational solutions with errors of less than $3 \%$.

The analytical solutions for the dissimilar aluminum/steel weld appear to be reasonably accurate. The analytical solutions have errors less than $3 \%$. Here, the effects of the clamped loading conditions on the stress intensity factor solutions based on the analytical solutions are examined. Table 13 lists the analytical normalized stress intensity factor $f_{1}, f_{2}$, and $f_{e}$ solutions for the dissimilar aluminum/steel weld in the specimen with $\delta=1$ under pinned and clamped loading conditions. As listed in the table, the bending moments and the transverse shear forces at the clamped edges reduce the stress intensity factor solutions by about 15 to $24 \%$ for the right crack and increase the stress intensity factor solutions by about 2 to $3 \%$ for the left crack for the dissimilar aluminum/steel weld. It appears that the effects of the clamped loading conditions can also be obtained from the analytical solutions when compared with the computational results listed in Table 3 for the dissimilar aluminum/steel weld.

Tables 14 to 16 list the normalized stress intensity factor solutions for the dissimilar magnesium/steel weld in the specimen with $\delta=1$ under pinned and clamped loading conditions. The computational results presented in Table 14 are in agreement with the results presented in Lai and Pan [6]. As listed in Tables 14 and 15, the analytical solutions for the dissimilar magnesium/steel weld appear to be reasonably accurate. As listed in Table 16, the bending moments and the transverse shear forces at the clamped edges reduce the stress intensity factor solutions by about 19 to $32 \%$ for the right crack and increase the stress intensity factor solutions by about 8 to $9 \%$ for the left crack for the dissimilar magnesium/steel weld. It appears that the effects of the clamped loading conditions can also be obtained from the analytical solutions when 
compared with the computational results listed in Table 4 for the dissimilar magnesium/steel weld.

Table 14. The normalized stress intensity factor $f_{1}^{p}, f_{2}^{p}$, and $f_{e}^{p}$ solutions for the dissimilar magnesium/steel weld in the specimen with $\delta=1$ under pinned loading conditions

\begin{tabular}{|c|c|c|c|c|c|c|}
\hline \multirow{2}{*}{ Pinned } & \multicolumn{3}{|c|}{ Left } & \multicolumn{3}{c|}{ Right } \\
\cline { 2 - 7 } & Analytical & Computational & Error & Analytical & Computational & Error \\
\hline$f_{1}^{p}$ & 0.5068 & 0.5077 & $0.18 \%$ & 1.0402 & 0.9854 & $-5.27 \%$ \\
\hline$f_{2}^{p}$ & 0.5239 & 0.5225 & $-0.27 \%$ & 1.4231 & 1.4606 & $2.64 \%$ \\
\hline$f_{e}^{p}$ & 0.7289 & 0.7285 & $-0.05 \%$ & 1.7627 & 1.7619 & $-0.05 \%$ \\
\hline
\end{tabular}

Table 15. The normalized stress intensity factor $f_{1}^{c}, f_{2}^{c}$, and $f_{e}^{c}$ solutions for the dissimilar magnesium/steel weld in the specimen with $\delta=1$ under clamped loading conditions

\begin{tabular}{|c|c|c|c|c|c|c|}
\hline \multirow{2}{*}{ Clamped } & \multicolumn{3}{|c|}{ Left } & \multicolumn{3}{c|}{ Right } \\
\cline { 2 - 7 } & Analytical & Computational & Error & Analytical & Computational & Error \\
\hline$f_{1}^{p}$ & 0.5523 & 0.5652 & $2.34 \%$ & 0.7088 & 0.6971 & $-1.65 \%$ \\
\hline$f_{2}^{p}$ & 0.5642 & 0.5668 & $0.46 \%$ & 1.1575 & 1.1915 & $2.94 \%$ \\
\hline$f_{e}^{p}$ & 0.7895 & 0.8004 & $1.38 \%$ & 1.3573 & 1.3804 & $1.70 \%$ \\
\hline
\end{tabular}

Table 16. The analytical normalized stress intensity factor $f_{1}, f_{2}$, and $f_{e}$ solutions for the dissimilar magnesium/steel weld in the specimen with $\delta=1$ under pinned and clamped loading conditions

\begin{tabular}{|c|c|c|c|c|c|c|}
\hline \multirow{2}{*}{ Analytical } & \multicolumn{3}{|c|}{ Left } & \multicolumn{3}{c|}{ Right } \\
\cline { 2 - 7 } & Pinned & Clamped & Change & Pinned & Clamped & Change \\
\hline$f_{1}$ & 0.5068 & 0.5523 & $+8.98 \%$ & 1.0402 & 0.7088 & $-31.86 \%$ \\
\hline$f_{2}$ & 0.5239 & 0.5642 & $+7.69 \%$ & 1.4231 & 1.1575 & $-18.66 \%$ \\
\hline$f_{e}$ & 0.7289 & 0.7895 & $+8.31 \%$ & 1.7627 & 1.3573 & $-23.00 \%$ \\
\hline
\end{tabular}


As discussed in Lai and Pan [6], the normalized transition weld length are defined at which the effective stress intensity factor solutions based on the beam theory is equal to the effective stress intensity factor solutions based on the small connection of two half planes. For the specimens of dissimilar material and equal thickness, the values of the normalized transition weld length were listed in Lai and Pan [6] for the dissimilar welds of magnesium, aluminum, steel and copper sheets. The values are between 0.209 for the dissimilar weld of magnesium and steel to 0.364 for the similar weld. The similar weld has the largest normalized transition weld length. The finite element analyses of Sripichai et al. [1] indicated that when the ratio of the weld length to sheet thickness is larger than 4 , the stress intensity factor solutions derived from the beam theory are applicable. As indicated by the values for the normalized transition weld length for similar and dissimilar welds in Lai and Pan [6], the stress intensity factor solutions for the welds in the specimens of dissimilar material and unequal thickness may also be applicable when the ratio of the weld length to sheet thickness is larger than 4 . The specimens investigated in this paper have the ratio of the weld length to sheet thickness of $5.07(=7.6 / 1.5)$. As indicated in the tables discussed earlier, the stress intensity factor solutions derived from the beam theory appear to be reasonably applicable.

The stress intensity factor solutions for the right cracks are larger than those for the left cracks for both the similar and dissimilar weld cases considered here mainly because the right cracks are associated with the thinner and less stiff (lower Young's modulus) sheets. For the dissimilar material cases, one can put a stiffer and thinner sheet as the upper sheet. Then the larger stress intensity factor solutions can be associated with the left crack as discussed for the transitional thickness ratio for dissimilar welds in Lai and Pan [6]. For the cases that are considered here, the stress intensity factor solutions for the right cracks for the similar and 
dissimilar cases under clamped loading conditions are smaller than those under pinned loading conditions from both the finite element analyses and analytical solutions. Consequently, the fatigue lives associated with the welds tested under clamped loading conditions should be higher than those under pinned loading conditions. Therefore, when the fatigue data for the welds in lap-shear specimens under cyclic loading conditions are to correlate with the stress intensity factor solutions for the welds, the loading conditions for the specimens should be carefully examined.

\section{Conclusions}

In this paper, the stress intensity factor solutions for similar and dissimilar welds in lap-shear specimens of equal and unequal thickness under clamped loading conditions are investigated. Finite element analyses are employed to obtain the stress intensity factor solutions for similar and dissimilar welds in lap-shear specimens under both pinned and clamped loading conditions. Analytical solutions for similar welds in lap-shear specimens of equal thickness are then derived based on the energy release rate approach. The analytical solutions show that the stress intensity factor solutions depend on the ratio of the weld length to specimen length due to the clamped loading conditions. For welds in lap-shear specimens of dissimilar material and unequal thickness, the analytical stress intensity factor solutions are obtained based on the structural stress approach. Both computational and analytical solutions indicate that the bending moments and the transverse shear forces at the clamped edges can reduce the stress intensity factor solutions for a given specimen geometry by about $7 \%$ for similar welds and by about $20 \%$ for dissimilar aluminum/steel and magnesium/steel welds. The analytical and computational stress intensity factor solutions suggest that the fatigue lives of the welds in lap-shear specimens under 
clamped loading conditions are higher than those under pinned loading conditions. Finally, all equations to obtain the stress intensity factor solutions are derived and provided in this paper for future applications where many combinations of materials, sheet thicknesses, weld lengths, and specimen lengths are possible for the lap-shear specimens under clamped loading conditions.

\section{Acknowledgement}

The initial support of this research by the U.S. Department of Energy is appreciated. Helpful discussions of this work with Seung Hoon Hong of University of Michigan and Shicheng Zhang of Daimler are greatly appreciated.

\section{References}

[1] Sripichai, K., Asim, K. and Pan, J., Stress intensity factor solutions for estimation of fatigue lives of laser welds in lap-shear specimens. Engineering Fracture Mechanics. 2011;78(7):14241440, doi:10.1016/j.engfracmech.2011.02.022.

[2] Asim, K., Sripichai, K. and Pan, J., Fatigue behavior of laser welds in lap-shear specimens of high strength low alloy steel sheets. International Journal of Fatigue. 2014;61:283-296, doi:10.1016/j.ijfatigue.2013.10.019.

[3] Cotterell, B. and Rice, J.R., Slightly curved or kinked cracks. International Journal of Fracture. 1980;16(2):155-169, doi:10.1007/BF00012619.

[4] Zhang, S., Stress intensities derived from stresses around a spot weld. International Journal of Fracture. 1999;99(4):239-257, doi: 10.1023/A:1018608615567.

[5] Suo, Z. and Hutchinson, J.W., Interface crack between two elastic layers. International Journal of Fracture. 1990;43(1):1-18, doi: 10.1007/BF00018123.

[6] Lai, W.-J. and Pan, J., Stress intensity factor solutions for dissimilar welds in lap-shear specimens of steel, magnesium, aluminum and copper sheets. SAE International Journal of Materials and Manufacturing. 2015;8(2), doi:10.4271/2015-01-1754.

[7] Hong, S.H., Sung, S.-J. and Pan, J., Failure mode and fatigue behavior of dissimilar friction stir spot welds in lap-shear specimens of transformation induced plasticity steel and hot stamped boron steel sheets. ASME Journal of Manufacturing Science and Engineering 2015;137(5):051023-051023-8, doi:10.1115/1.4031235. 
[8] Sung, S.-J. and Pan, J., Stress intensity factor solutions for welds in lap-shear specimens under clamped loading conditions. SAE International Journal of Materials and Manufacturing. 2016;9(3), doi:10.4271/2016-01-0504.

[9] Lin, P.-C. and Pan, J., Closed-form structural stress and stress intensity factor solutions for spot welds in commonly used specimens. Engineering Fracture Mechanics. 2008;75(18):51875206, doi: 10.1016/j.engfracmech.2008.08.005.

[10] Tran, V.-X. and Pan, J., Analytical stress intensity factor solutions for resistance and friction stir spot welds in lap-shear specimens of different materials and thicknesses. Engineering Fracture Mechanics. 2010;77(14):2611-2639, doi: 10.1016/j.engfracmech.2010.06.022.

[11] Rice, J.R. and Sih, G.C., Plane problems of cracks in dissimilar media. Journal of Applied Mechanics. 1965;32(2):418-423, doi: 10.1115/1.3625816.

[12] Rice, J.R., Elastic fracture mechanics concepts for interfacial cracks. Journal of Applied Mechanics. 1988;55(1):98-103, doi: 10.1115/1.3173668.

[13] Pook, L.P., Approximate stress intensity factors obtained from simple plane bending theory. Engineering Fracture Mechanics. 1979;12(4):505-522, doi: 10.1016/0013-7944(79)90093-6.

[14] Rice, J.R., A path independent integral and the approximate analysis of strain concentration by notches and cracks. Journal of Applied Mechanics. 1968;35(2):379-386, doi:

10.1115/1.3601206. 


\section{Appendix A: Derivation of Stress Intensity Factor Solutions for Specimen of Similar Material and Equal Thickness}

For linear elastic materials, the stress intensity factor solutions can be derived from the energy release rate $G$ which represents the decrease of the potential energy per unit crack area growth. The decrease of the potential energy per unit crack area growth is equal to the increase of the elastic energy per crack area growth for linear elastic materials. The use of the J integral approach in Zhang [4] can also result in the same results. Under plane strain conditions, the relationship between the energy release rate $G$ and the stress intensity factors can be expressed as

$$
G=\frac{d U}{b d x}=\frac{K_{I}^{2}+K_{I I}^{2}}{E^{\prime}}
$$

where $d U$ is the increase of the elastic strain energy, $b$ is the specimen width, and $d x$ is the increase of the crack length. Here, $E^{\prime}=E /\left(1-v^{2}\right)$ where $E$ is the Young's modulus and $v$ is the Poisson's ratio.

Figure 17(a) shows the beam model under the load at E. As shown in the figure, the original load can be decomposed into the loads that give the counter bending, central bending, tension and in-plane shear loading conditions as shown in Figures 17(b), 17(c), 17(d), and 17(e), respectively. The energy release rate for the crack area growth $b d x$ and the stress intensity factor for each of the loading conditions will be discussed in the following. 


\section{A1. Counter bending condition}

Figure 17(b) shows the beam model under counter bending condition. Note that the symmetric loading gives $K_{I}$. The elastic energy increase $d U$ for the unit crack area growth $b d x$ is

$$
d U=2\left(\frac{1}{2} \frac{\left(\frac{M_{E}}{2}\right)^{2}}{E^{\prime} \frac{b t^{3}}{12}}\right) b d x
$$

Simplifying Equation (A2) gives the energy release rate $G$ as

$$
G=\frac{d U}{b d x}=3 \frac{M_{E}^{2}}{E^{\prime} b t^{3}}=\frac{K_{I}^{2}}{E^{\prime}}
$$

With the solution of $M_{E}, K_{I}$ can be obtained as

$$
K_{I}=\left(\frac{\sqrt{3}}{2}-\frac{3 \sqrt{3}}{4} \frac{r}{L} \lambda\right) \frac{F}{b \sqrt{t}}
$$

Here, $\lambda$ is a function of $r / L$ and $\lambda$ is defined in Equation (42).

\section{A2. Central bending condition}

Figure 17(c) shows the beam model under central bending condition. Note that the antisymmetric loading gives $K_{I I}$. The elastic energy increase $d U$ for the unit crack area growth $b d x$ is

$$
d U=\left(\frac{1}{2} \frac{\left(\frac{M_{E}}{2}\right)^{2}}{E^{\prime} \frac{b t^{3}}{12}} 2-\frac{1}{2} \frac{\left(M_{E}\right)^{2}}{E^{\prime} \frac{b(2 t)^{3}}{12}}\right) b d x
$$


Simplifying Equation (A5) gives the energy release rate $G$ as

$$
G=\frac{d U}{b d x}=\frac{9}{4} \frac{M_{E}^{2}}{E^{\prime} b t^{3}}=\frac{K_{I I}^{2}}{E^{\prime}}
$$

With the solution for $M_{E}, K_{I I}$ is

$$
K_{I I}=\left(\frac{3}{4}-\frac{9}{8} \frac{r}{L} \lambda\right) \frac{F}{b \sqrt{t}}
$$

\section{A3. In-plane shear loading condition}

Figure 17(e) shows the beam model under in-plane shear loading condition. Note that the anti-symmetric loading gives $K_{I I}$. The elastic energy increase $d U$ for the unit crack area growth $b d x$ is

$$
d U=\left(2 \frac{1}{2} \frac{\left(\frac{F}{2}\right)^{2}}{E^{\prime} b t}-\frac{1}{2} \frac{\left(\frac{F t}{2}\right)^{2}}{E^{\prime} \frac{b(2 t)^{3}}{12}}\right) b d x
$$

Simplifying Equation (A8) gives the energy release rate $G$ as

$$
G=\frac{d U}{b d x}=\frac{1}{16} \frac{F^{2}}{E^{\prime} b^{2} t}=\frac{K_{I I}^{2}}{E^{\prime}}
$$

Therefore, $K_{I I}$ is

$$
K_{I I}=\frac{1}{4} \frac{F}{b \sqrt{t}}
$$


A4. Stress intensity factor solutions under lap-shear loading conditions

Based on the superposition principle of the linear elasticity, the total stress intensity factor solutions are the combinations of the stress intensity factor solutions from the counter bending, central bending and in-plane shear loading conditions. The tension loading condition does not induce any stress intensity factor. $K_{I}$ and $K_{I I}$ are now expressed as

$$
K_{I}=\left(\frac{\sqrt{3}}{2}-\frac{3 \sqrt{3}}{4} \frac{r}{L} \lambda\right) \frac{F}{b \sqrt{t}}
$$

and

$$
K_{I I}=\left(1-\frac{9}{8} \frac{r}{L} \lambda\right) \frac{F}{b \sqrt{t}}
$$

